\title{
Feedback cooling of atomic motion in cavity QED
}

\author{
Daniel A. Steck, ${ }^{1,2}$ Kurt Jacobs, ${ }^{1,3,4}$ Hideo Mabuchi, ${ }^{5}$ Salman Habib, ${ }^{1}$ and Tanmoy Bhattacharya ${ }^{1}$ \\ ${ }^{1}$ Theoretical Division (T-8), MS B285, Los Alamos National Laboratory, Los Alamos, New Mexico 87545, USA \\ ${ }^{2}$ Oregon Center for Optics and Department of Physics, 1274 University of Oregon, Eugene, Oregon 97403-1274, USA \\ ${ }^{3}$ Centre for Quantum Computer Technology, Centre for Quantum Dynamics, School of Science, Griffith University, \\ Nathan 4111, Australia \\ ${ }^{4}$ Quantum Science and Technologies Group, Hearne Institute for Theoretical Physics, Department of Physics and Astronomy, \\ Louisiana State University, 202 Nicholson Hall, Tower Drive, Baton Rouge, Louisiana 70803, USA \\ ${ }^{5}$ Norman Bridge Laboratory of Physics 12-33, California Institute of Technology, Pasadena, California 91125, USA
}

(Received 8 October 2005; published 31 July 2006)

\begin{abstract}
We consider the problem of controlling the motion of an atom trapped in an optical cavity using continuous feedback. In order to realize such a scheme experimentally, one must be able to perform state estimation of the atomic motion in real time. While in theory this estimate may be provided by a stochastic master equation describing the full dynamics of the observed system, integrating this equation in real time is impractical. Here we derive an approximate estimation equation for this purpose, and use it as a drive in a feedback algorithm designed to cool the motion of the atom. We examine the effectiveness of such a procedure using full simulations of the cavity QED system, including the quantized motion of the atom in one dimension.
\end{abstract}

DOI: 10.1103/PhysRevA.74.012322

PACS number(s): 03.67.-a, 02.30.Yy, 32.80.Pj, 42.50.-p

\section{INTRODUCTION}

The theory of quantum measurement has been mired in controversy for the majority of its history. In a large part this is due to the philosophical difficulties, usually referred to as the "quantum measurement problem," with which it is associated [1]. However, in most areas of physics it has been generally possible to ignore quantum measurement theory, because it was largely irrelevant to real measurements that were being made by experimentalists [2]. In particular, if needed at all, an ensemble picture in which quantum measurement theory merely predicted average statistics provided a sufficient description. However, experimental technology has now advanced to the point where repeated measurements may be made on a single quantum system as it evolves [3-6]. As a result, not only does quantum measurement theory become directly relevant to experimental physics, but we can consider testing the predictions of this theory regarding the effects of measurement on the single system itself.

While the theory of quantum measurement is now widely accepted, some still remain unconvinced that the conditioned state describes what is really happening to an individual system when it is measured. How might we verify these predictions, and bring at least this part of the controversy to an end? One way to do this is to attempt to perform feedback control on an individual quantum system $[7,8]$. Such an experiment involves taking into account the changes in the system due to the measurement results, and using this knowledge to alter inputs to the system in order to control its dynamics. This would therefore provide a direct verification of quantum measurement theory: if the feedback algorithm works, then the theory is correctly predicting the behavior of the system; conversely, if the theory is not giving accurate predictions, the feedback algorithm will be ineffective.

Cavity quantum electrodynamics (CQED) is one area in which the motion of a single quantum system, an individual atom in an optical cavity, can be monitored experimentally in real time [3-6,9]. Not only does the light emitted from the optical cavity provide a means to track the atomic motion, but the laser driving the cavity provides a means to apply forces to the atom. This system is therefore an excellent candidate for implementing real-time quantum feedback control. It is our purpose here to examine the implementation of such a process in this system, and to show that by using approximate estimation techniques, which will be essential in such experiments for the foreseeable future, a measurable degree of control over the atomic motion can be realized. In particular, we will simulate a control algorithm designed to cool the atomic motion. This might be referred to as "active" cooling (control) [7,10-33], as opposed to passive cooling schemes which have also been the focus of recent theoretical [34-42] and experimental [43] work.

In general, feedback control of the atomic motional state requires the real-time estimation of the quantum state from the continuous measurement, thus providing the information needed to decide how the system should be perturbed to bring it to the target state. The theoretical tools required for obtaining this continuous estimate are those of continuous quantum measurement $[8,44-56]$. An estimate of the state of the quantum system (in our case the motional state of the atom), conditioned continuously upon the results of measurement, is given by a stochastic master equation (SME). The concept of an SME was first developed by Belavkin in Ref. [8] (as the quantum equivalent of the classical KushnerStratonovich equation), and a derivation of an SME in the language of quantum optics may be found in Ref. [56]. In principle, the observer may calculate this state estimate by integrating the relevant SME using the measurement results, and from this determine the values of the inputs to the system to effect control. However, in practice the complexity of the SME will prevent the observer from calculating the state estimate in real time for systems where the dynamics are sufficiently fast, such as the atomic dynamics we consider here. As a result, a simplified state-estimation procedure is essential. 
The theory of feedback control for classically observed linear systems is well developed [57-60]. This theory considers the optimality and robustness of feedback algorithms for given resources, and in some special cases may be applied directly to the control of quantum systems $[8,12]$. Discussions of the application of classical feedback control techniques to quantum systems may be found in Refs. [11-13]. However, for nonlinear control problems $[13,61,62]$, such as that of the atomic motion we consider, no general results regarding the optimality of control algorithms exist as of yet. Here we will be concerned with presenting simple control algorithms for cooling the atoms, and demonstrating that along with an approximate estimation algorithm, a substantial intervention into the dynamics of the atom using realtime feedback control should be achievable experimentally in the near future. In doing so, we build upon previous results [30] by providing a detailed analysis of the cooling performance in this system, including its robustness to parameter variations and real-world limitations on computational speed and detection efficiency. Furthermore, we are able to anticipate several difficulties in an experimental implementation and suggest solutions to overcome them.

Owing to the computationally expensive nature of simulating an atom in an optical cavity, including the atomic motion in one dimension, supercomputers are invaluable for the task of evaluating the performance of the control algorithm over many realizations. We have performed full quantum simulations of such a CQED system, including the quantized, one-dimensional motion of the atom. We are thus able to confirm the results of various approximations which have been used previously to provide a simplified analysis of this system [12,63-66].

\section{DESCRIPTION OF THE SYSTEM}

The system we consider here is that of a two-level atom in a driven, single-mode optical cavity, where the output from the cavity is monitored using homodyne detection [55,56]. Due to the continuous observation, the evolution of the system is described by a stochastic master equation (SME) for the density operator, which can be written in Itô form as $[67,73,74]$

$$
\begin{aligned}
d \rho= & -i[(H / \hbar), \rho] d t+\gamma d t \int_{-\hbar k}^{\hbar k} N(u) \mathcal{D}\left[\sigma e^{-i u x / \hbar}\right] \rho d u \\
& +\kappa \mathcal{D}[a] \rho d t+\sqrt{\eta \kappa} \mathcal{H}[a] \rho d W,
\end{aligned}
$$

where the Hamiltonian is [66]

$$
\frac{H}{\hbar}=\frac{p^{2}}{2 m \hbar}+g \cos (k x)\left(\sigma^{\dagger} a e^{i \Delta t}+\text { H.c. }\right)-E\left(a+a^{\dagger}\right),
$$

and we have defined the superoperators $\mathcal{D}[c]$ and $\mathcal{H}[c]$ by

$$
\begin{gathered}
\mathcal{D}[c] \rho:=c \rho c^{\dagger}-\frac{1}{2}\left(c^{\dagger} c \rho+\rho c^{\dagger} c\right), \\
\mathcal{H}[c] \rho:=c \rho+\rho c^{\dagger}-\left\langle c+c^{\dagger}\right\rangle \rho
\end{gathered}
$$

for an arbitrary operator $c$. In addition, the measured homodyne signal, given by

$$
d r(t)=\eta \kappa\left\langle a+a^{\dagger}\right\rangle d t+\sqrt{\eta \kappa} d W,
$$

continuously provides information about the optical phase shift due to the atom-cavity system and hence, as we will see below, information about the atomic position. In the above equations, $x$ is an operator representing the atomic position along the length of the cavity, $p$ is the conjugate momentum operator, $a$ is the annihilation operator for the cavity mode, and $\sigma$ is the lowering operator for the atomic internal states. The energy loss rate of the cavity (or equivalently, the fullwidth-at-half-maximum cavity transmission) is $\kappa$, and $E$ gives the strength of the driving laser, and is related to the laser power $P$ by $E=\sqrt{\kappa P /\left(\hbar \omega_{\mathrm{L}}\right)}$, where $\omega_{\mathrm{L}}$ is the angular frequency of the laser light. We take the laser frequency to be resonant with the cavity mode. The laser angular wave number is given by $k=\omega_{\mathrm{L}} / c$, the decay rate of the atomic excited state is denoted by $\gamma, g$ is the CQED coupling constant between the atomic internal states and the cavity mode, and $\Delta$ is the detuning between the atom and the cavity mode, given by $\Delta=-\left(\omega_{\mathrm{L}}-\omega_{\mathrm{A}}\right)$, where $\omega_{\mathrm{A}}$ is the atomic transition frequency. We will assume a positive (red) detuning, corresponding to a trapping optical potential. Also, $N(u)$ is the probability that the atomic momentum recoil along the $x$ direction is $u$ due to a spontaneous emission event, and $d W$ is the differential of a stochastic Wiener process. Note that we have written the stochastic master equation in the interaction picture where the free oscillation of the light and the dipole rotation of the atom are implicit.

The density operator $\rho(t)$ obtained as a solution to the SME condenses our knowledge of the initial condition and the measurement record, allowing a prediction of the statistics of any future measurement on the system. As we show numerically in Sec. IV C, the solution to the equation is, generically, insensitive to the initial conditions except at very early times. We therefore refer to the solution of the SME as the observer's "best estimate" or "state of knowledge" of the quantum system.

In order to perform numerical calculations, it is sensible to choose units for position, momentum, and time that are relevant for the corresponding scales of the system. When the detuning of the atom from the cavity is large compared to the cavity decay rate and the CQED coupling constant, both the internal atomic states and the cavity mode may be adiabatically eliminated, and in this case the atom sees an effective potential, given by

$$
V_{\text {eff }}(x)=-\hbar \frac{g^{2} \alpha^{2}}{\Delta} \cos ^{2}(k x),
$$

where $\alpha:=2 E / \kappa$. Since this regime is the one in which we will be primarily interested, convenient units for $x$ and $p$ are given respectively by the width in position and momentum of the ground-state wave function in the harmonic approximation to one of the cosine wells. A convenient unit for time is set by the frequency of oscillation in the same harmonic potential. Thus, given the three scales of the problem, 


$$
\begin{gathered}
\omega_{\mathrm{HO}}:=\alpha g k \sqrt{2 \hbar /|m \Delta|}, \\
w_{x}:=\sqrt{\hbar /\left(m \omega_{\mathrm{HO}}\right)}, \\
w_{p}:=\sqrt{\hbar m \omega_{\mathrm{HO}}},
\end{gathered}
$$

we will use the scaled variables $X:=x / w_{x}$ and $P:=p / w_{p}$, and we will measure time in units of $2 \pi / \omega_{\mathrm{HO}}$. The SME for the density matrix may now be written as

$$
\begin{aligned}
d \rho= & -i[H, \rho] d t+\tilde{\gamma} d t \int_{-\tilde{k}}^{\tilde{k}} N(\tilde{u}) \mathcal{D}\left[\sigma e^{-i \tilde{u} X}\right] \rho d \tilde{u}+\tilde{\kappa} \mathcal{D}[a] \rho d t \\
& +\sqrt{\eta \tilde{\kappa}} \mathcal{H}[a] d W
\end{aligned}
$$

where

$$
H=\pi P^{2}+\tilde{g} \cos (\tilde{k} X)\left(\sigma^{\dagger} a e^{i \tilde{\Delta} t}+\text { H.c. }\right)-\widetilde{E}\left(a+a^{\dagger}\right)
$$

and

$$
d \widetilde{r}(t)=\eta\left\langle a+a^{\dagger}\right\rangle d t+\sqrt{\eta} d W
$$

with $d \widetilde{r}(t)=d r(t) / \sqrt{\widetilde{\kappa}}$. The scaled rate constants $\widetilde{\kappa}, \widetilde{E}, \widetilde{\Delta}, \widetilde{\gamma}$, and $\tilde{g}=\sqrt{\pi \tilde{\Delta}} /(\alpha \tilde{k})$ are obtained from their absolute counterparts by dividing by $\omega_{\mathrm{HO}} /(2 \pi)$. The scaled wave number is naturally $\tilde{k}=k w_{x}$. Note that in these scaled units we have scaled $\hbar$ out of the problem, so that $[X, P]=i$.

While we will solve this equation numerically, it is nevertheless useful to examine the solutions obtained by adiabatically eliminating both the atomic internal states and the cavity mode, which is possible when the detuning $\widetilde{\Delta}$ is much larger than the coupling constant $\tilde{g}$ so that the excited atomic state is nearly unpopulated during the evolution [66]. In this case the Hamiltonian part of the evolution is governed by the effective Hamiltonian

$$
H_{\mathrm{eff}}:=\pi P^{2}-\frac{\widetilde{g}^{2}}{\widetilde{\Delta}} \cos ^{2}(\tilde{k} X) a^{\dagger} a .
$$

Thus, the atom moves in a sinusoidal potential, with period $\pi / \tilde{k}$. The average height of the potential wells is essentially proportional to $\left\langle a^{\dagger} a\right\rangle$. It is therefore instructive to consider the cavity-mode dynamics, which determine the magnitude of the effective potential. Under the effective Hamiltonian, the Heisenberg equations of motion for the relevant cavity mode operators are

$$
\begin{gathered}
\partial_{t} a=i \widetilde{E}-\frac{\widetilde{\kappa}}{2} a+i \frac{\widetilde{g}^{2}}{\widetilde{\Delta}} \cos ^{2}(\widetilde{k} X) a+\sqrt{\widetilde{\kappa}} a_{\mathrm{in}}(t), \\
\partial_{t}\left(a^{\dagger} a\right)=-\widetilde{\kappa} a^{\dagger} a-i \widetilde{E}\left(a-a^{\dagger}\right)+\sqrt{\widetilde{\kappa}}\left[a^{\dagger} a_{\mathrm{in}}(t)+a_{\mathrm{in}}^{\dagger}(t) a\right],
\end{gathered}
$$

where $a_{\text {in }}(t)$ is the input quantum noise operator coming from the electromagnetic field outside the cavity, and satisfies $\left[a_{\text {in }}(t), a_{\text {in }}^{\dagger}(t+\tau)\right]=\delta(\tau)$. We can now identify a regime in which $\widetilde{\kappa}$ is large compared to $\widetilde{g}^{2} / \widetilde{\Delta}$. In this case the cavity mode damps quickly to a steady state, and we can approximate the solution by

$$
\begin{gathered}
a \approx i \alpha\left[1-i 2\left(\frac{\widetilde{g}^{2}}{\widetilde{\kappa} \widetilde{\Delta}}\right) \cos ^{2}(\tilde{k} X)\right]^{-1}, \\
\approx i \alpha-\alpha\left(\frac{2 \widetilde{g}^{2}}{\widetilde{\Delta} \widetilde{\kappa}}\right) \cos ^{2}(\widetilde{k} X), \\
a^{\dagger} a \approx \alpha \frac{\left(a-a^{\dagger}\right)}{2 i} .
\end{gathered}
$$

There will be fluctuations about these values due to the noise with a spectrum with a width of the order of $\tilde{\kappa}$. When $\kappa$ (or $\widetilde{\kappa})$ is sufficiently large, then $\left\langle a^{\dagger} a\right\rangle \approx \alpha^{2}$, and the height of the potential will therefore be determined by the strength of the driving. As a consequence, one can manipulate the potential that the atom sees by changing the strength $\widetilde{E}$ of the driving. The measured homodyne signal is given in the adiabatic approximation by

$$
d \widetilde{r}(t)=-\sqrt{8 \eta^{2} \Gamma}\left\langle\cos ^{2}(\tilde{k} X)\right\rangle d t+\sqrt{\eta} d W,
$$

where $d \widetilde{r}(t):=d r(t) / \sqrt{\widetilde{\kappa}}$; the position-information content of this signal is more clear in this form.

\section{COOLING THE ATOMIC MOTION USING FEEDBACK}

As mentioned in the Introduction, in implementing feedback control, two considerations are paramount. The first is the ability to effectively track the evolution of the system in real time (state estimation). The second is to provide an algorithm that determines how this information about the system should be used to alter the inputs to the system to effect control. We now consider these two questions in turn.

\section{A. State estimation in real time}

The SME given in Eq. (1) continuously provides the observer's best estimate of the state of the system from the information provided by the measurement record $r(t)$ [Eq. (4)]. Therefore, it is possible, in principle, to use the measurement record to integrate the full SME to obtain a continuous state estimate. However, the resources required to do this in real time are prohibitive in practice. This is because the SME, being a partial stochastic differential equation, contains a large effective number of variables, and is almost impossible to integrate in real time, given that the time scale of the atomic motion is typically a few $\mu$ s. As a result, we must obtain an estimation equation which contains only a small number of variables, but is nevertheless a good approximation to the full SME.

To obtain a compact system of estimation equations, we first note that initially Gaussian states remain close to Gaussian for a fairly large number of oscillations in a sufficiently strong sinusoidal potential. We thus make a Gaussian approximation for the density matrix, which amounts to ignor- 
ing all cumulants higher than second order. In the present system, which effectively measures $\left\langle\cos ^{2}(\tilde{k} X)\right\rangle$, we expect this approximation to remain valid as long as the wave packet is much narrower than a single well $(\tilde{k} \Delta X \ll 1)$, and the wave-packet momentum width is much larger than two photon recoils $(\Delta P \gg 2 \widetilde{k})$. To further simplify the estimation equations, we consider the SME after adiabatic elimination of the cavity and internal atomic degrees of freedom, given by

$$
\begin{aligned}
d \rho= & -i\left[H_{\mathrm{eff}}, \rho\right] d t+2 \Gamma \mathcal{D}\left[\cos ^{2}(\tilde{k} X)\right] \rho d t \\
& -\sqrt{2 \eta \Gamma} \mathcal{H}\left[\cos ^{2}(\tilde{k} X)\right] \rho d W,
\end{aligned}
$$

which gives a three-parameter model $(\widetilde{k}, \Gamma$, and $\eta)$. Here, we have defined the effective Hamiltonian

$$
H_{\mathrm{eff}}=\pi P^{2}-V_{\max } \cos ^{2}(\tilde{k} X),
$$

where the maximum light shift

$$
V_{\max }:=\frac{\alpha^{2} \widetilde{g}^{2}}{\widetilde{\Delta}}=\frac{\pi}{\widetilde{k}^{2}}
$$

is not an independent parameter in this static problem, but we will treat it as such when considering feedback via a time-dependent potential. We have also defined the effective measurement strength

$$
\Gamma:=\frac{2 \alpha^{2} \widetilde{g}^{4}}{\widetilde{\Delta}^{2} \widetilde{\kappa}} .
$$

We further simplify the estimation model by making a Gaussian ansatz for the density operator. The resulting estimation equations contain merely five variables: the estimated mean position and momentum $\langle X\rangle_{\mathrm{e}}$ and $\langle P\rangle_{\mathrm{e}}$, the estimated variances $V_{X}^{\mathrm{e}}$ and $V_{P}^{\mathrm{e}}$, and the estimated symmetrized covariance $C_{\mathrm{e}} \mathrm{:}=\langle X P+P X\rangle_{\mathrm{e}} / 2-\langle X\rangle_{\mathrm{e}}\langle P\rangle_{\mathrm{e}}$. (Due to the information content of the measurement process, the two variances and the covariance are algebraically independent, in contrast to a closed system.) Using the equation of motion for the expectation value of an arbitrary operator $A$,

$$
\begin{aligned}
d\langle A\rangle= & \operatorname{Tr}[A d \rho]=-i\left\langle\left[A, H_{\text {eff }}\right]\right\rangle d t+2 \Gamma\left\langle D\left[\cos ^{2}(\tilde{k} X)\right] A\right\rangle d t \\
& -\sqrt{2 \eta \Gamma}\left\langle\mathcal{H}\left[\cos ^{2}(\tilde{k} X)\right] A\right\rangle d W,
\end{aligned}
$$

we can calculate the system of five Gaussian estimation equations, which are

$$
\begin{gathered}
d\langle X\rangle_{\mathrm{e}}=2 \pi\langle P\rangle_{\mathrm{e}} d t+\sqrt{8 \eta \Gamma} \widetilde{k} V_{X}^{\mathrm{e}} \exp \left(-2 \widetilde{k}^{2} V_{X}^{\mathrm{e}}\right) \sin \left(2 \widetilde{k}\langle X\rangle_{\mathrm{e}}\right) d W_{\mathrm{e}}, \\
d\langle P\rangle_{\mathrm{e}}=-V_{\max } \widetilde{k} \exp \left(-2 \widetilde{k}^{2} V_{X}^{\mathrm{e}}\right) \sin \left(2 \widetilde{k}\langle X\rangle_{\mathrm{e}}\right) d t+\sqrt{8 \eta \Gamma} \widetilde{k} C_{\mathrm{e}} \exp \left(-2 \widetilde{k}^{2} V_{X}^{\mathrm{e}}\right) \sin \left(2 \widetilde{k}\langle X\rangle_{\mathrm{e}}\right) d W_{\mathrm{e}}, \\
d V_{X}^{\mathrm{e}}=4 \pi C_{\mathrm{e}} d t-8 \eta \Gamma \widetilde{k}^{2} V_{X}^{\mathrm{e} 2} \exp \left(-4 \widetilde{k}^{2} V_{X}^{\mathrm{e}}\right) \sin ^{2}\left(2 \widetilde{k}\langle X\rangle_{\mathrm{e}}\right) d t+2 \sqrt{8 \eta \Gamma} \widetilde{k}^{2} V_{X}^{\mathrm{e} 2} \exp \left(-2 \widetilde{k}^{2} V_{X}^{\mathrm{e}}\right) \cos \left(2 \widetilde{k}\langle X\rangle_{\mathrm{e}}\right) d W_{\mathrm{e}}, \\
d V_{P}^{\mathrm{e}}=-4 V_{\mathrm{max}} \widetilde{k}^{2} C_{\mathrm{e}} \exp \left(-2 \widetilde{k}^{2} V_{X}^{\mathrm{e}}\right) \cos \left(2 \widetilde{k}\langle X\rangle_{\mathrm{e}}\right) d t+\Gamma \widetilde{k}^{2}\left[1-\exp \left(-8 \widetilde{k}^{2} V_{X}^{\mathrm{e}}\right) \cos \left(4 \widetilde{k}\langle X\rangle_{\mathrm{e}}\right)\right] d t-8 \eta \Gamma \widetilde{k}^{2} C_{\mathrm{e}}^{2} \exp \left(-4 \widetilde{k}^{2} V_{X}^{\mathrm{e}}\right) \sin ^{2}\left(2 \widetilde{k}\langle X\rangle_{\mathrm{e}}\right) d t \\
-\sqrt{2 \eta \Gamma} \widetilde{k}^{2}\left[1-4\left(C_{\mathrm{e}}^{2}+\widetilde{k}^{2} V_{X}^{\mathrm{e}}\right) \exp \left(-2 \widetilde{k}^{2} V_{X}^{\mathrm{e}}\right)\right] \cos \left(2 \widetilde{k}\langle X\rangle_{\mathrm{e}}\right) d W_{\mathrm{e}}, \\
d C_{\mathrm{e}}=2 \pi V_{P}^{\mathrm{e}} d t-2 V_{\max } \widetilde{k}^{2} V_{X}^{\mathrm{e}} \exp \left(-2 \widetilde{k}^{2} V_{X}^{\mathrm{e}}\right) \cos \left(2 \widetilde{k}\langle X\rangle_{\mathrm{e}}\right) d t-8 \eta \Gamma \widetilde{k}^{2} V_{X}^{\mathrm{e}} C_{\mathrm{e}} \exp \left(-4 \widetilde{k}^{2} V_{X}^{\mathrm{e}}\right) \sin ^{2}\left(2 \widetilde{k}\langle X\rangle_{\mathrm{e}}\right) d t \\
+2 \sqrt{8 \eta \Gamma} \widetilde{k}^{2} V_{X}^{\mathrm{e}} C_{\mathrm{e}} \exp \left(-2 \widetilde{k}^{2} V_{X}^{\mathrm{e}}\right) \cos \left(2 \widetilde{k}\langle X\rangle_{\mathrm{e}}\right) d W_{\mathrm{e}} .
\end{gathered}
$$

Although we can eliminate $V_{\max }$ from these estimator equations (since $V_{\max } \widetilde{k}^{2}=\pi$ ), we keep the optical potential explicit here because both $V_{\max }$ and $\Gamma$ will effectively become timedependent quantities when we consider feedback control by modulating the amplitude of the optical potential. The reconstructed "Wiener increment" $d W_{\mathrm{e}}$ arises by making the Gaussian approximation to the photocurrent equation (13)

$d W_{\mathrm{e}}=\frac{d \widetilde{r}(t)}{\sqrt{\eta}}+\sqrt{2 \eta \Gamma}\left[1+\exp \left(-2 \widetilde{k}^{2} V_{X}^{\mathrm{e}}\right) \cos \left(2 \widetilde{k}\langle X\rangle_{\mathrm{e}}\right)\right] d t$.
These estimation equations resemble stochastic differential equations, but it is important to recognize that $d W_{\mathrm{e}}$ does not have the same statistics as the usual Wiener increment $d W$. In particular, the reconstructed increment is the usual Wiener increment with an extra "deterministic" component

$$
\begin{aligned}
d W_{\mathrm{e}}= & d W+\sqrt{2 \eta \Gamma}\left[\exp \left(-2 \widetilde{k}^{2} V_{X}^{\mathrm{e}}\right) \cos \left(2 \tilde{k}\langle X\rangle_{\mathrm{e}}\right)\right. \\
& -\langle\cos (2 \tilde{k} X)\rangle] d t,
\end{aligned}
$$

which reflects the difference between the actual and estimated quantum states. This is the route by which the measurement information is incorporated into the estimator evo- 
lution. Thus, these equations cannot be treated by the usual high-order numerical methods for stochastic differential equations, which assume that the deterministic and stochastic parts of the differential equation can be explicitly separated.

Another important issue to note is that because the estimator only has information about $\left\langle\cos ^{2}(\widetilde{k} X)\right\rangle$, it cannot tell us in which well the atom is located, and therefore, while feedback will allow us to cool an atom within a well, we cannot know within which well the atom is confined. Moreover, the estimator cannot tell us on what side of a well the atom is located. Note that this degeneracy is not a consequence of the Gaussian approximation, but also applies to an observer using the full SME for estimation. As a consequence, the estimator will show us the motion of the atom up to a phase factor of $\pi$ : the estimated state will either be in phase with the true motion, or completely out of phase, since these two motions are mirror images of one another. Thus, for the feedback algorithm to be effective for every run, it is important that it works for both cases without change. The algorithms that we describe in the next section have this property.

It is worth noting that the insensitivity of the feedback algorithm to the side of the well the atom is on has a potential advantage. In simulating the dynamics of the atom, we find that if the initial energy of the atom is large enough for it to move from one well to another, then as it does so the wave function tends to split across the interwell barrier, with part going back down into the original well, and part going down into an adjacent well. In this case the wave function is no longer Gaussian, but consists of two or more "humps." However, even though the two humps are on opposite sides of their respective wells, they have approximately the same downward motion. Thus, even though the estimator is Gaussian, the fact that the humps are in different wells is immaterial to the measurement of $\left\langle\cos ^{2}(\widetilde{k} X)\right\rangle$, and the estimator effectively tracks the evolution of an "equivalent" quantum state localized within a single well in the sense of generating the same measurement record as the fragmented state. As a result, we can expect the Gaussian estimator to continue to work even though the atom is distributed across more than one well.

In order to perform the continuous estimation (whether using the full SME or the above Gaussian approximate estimator) one chooses an initial state for the estimator that reflects the initial ignorance of the true quantum state and integrates the estimator equations using the measurement record as this record is obtained. In our case, when the atom is dropped into the cavity we have very little idea of where it is. It is therefore sensible to choose as the initial state for the estimator a Gaussian broad enough to cover one side of a well, and centered somewhere on that side (it is not important where precisely). We only need to include one side of the well in our initial uncertainty, since as mentioned above, both sides are equivalent from the point of view of the estimator. In practice, though, it is sufficient to pick a state with a much smaller uncertainty product, centered on one side of a potential well. Even if this estimator state does not substantially overlap the actual quantum state, as we will see below, it will converge to the "true" state in a time of order $1 / \Gamma$ as the measurement information is incorporated.

\section{B. An effective cooling algorithm}

As discussed in Sec. II, it is possible to raise and lower the height of the potential by changing the input laser power. Thinking of the atom as a classical particle, one would expect to be able to cool the atom using the following simple algorithm: When the atom is moving toward the center of the well, it is being accelerated by the potential, and so we can lower the laser power to reduce this acceleration. On the other hand, when the atom is moving away from the center of the well, then the potential decelerates the atom, and in this case we can increase the laser power so as to increase the deceleration. Writing the optical field amplitude in the absence of feedback as $E$, we will denote the value of the field during times when it is "switched high" as $\left(1+\varepsilon_{1}\right) E$ and the value when it is "switched low" as $\left(1-\varepsilon_{2}\right) E$. Thus the potential height will have the respective values $\left(1+\varepsilon_{1}\right)^{2} V$ and $(1$ $\left.-\varepsilon_{2}\right)^{2} V$, where $V$ is the unmodulated potential depth. In this way, we repeatedly switch the potential between a high value and a low value, which both slows the atom and moves it in towards center of the well, reducing the total energy and hence cooling the atom. This algorithm also has the nice property that all the information it requires is whether the atom is climbing or descending the well. Therefore, the question of whether the estimated state is in phase or completely out of phase with the true motion is immaterial, since in both cases the relevant information is known. However, it turns out that this algorithm is not very effective at cooling the atomic motion, because while it does cool the centroid, it simultaneously feeds energy into the motion by squeezing the atomic wave function. A detailed discussion of this effect is given in Appendix A. The result is that this simple centroid-only cooling algorithm is not adequate to cool the atom to the ground state. To do so we must develop a more sophisticated algorithm which can reduce the motional energy associated with the variances of the wave function.

To proceed, we consider the change in the motional energy due to time dependence of the optical-potential amplitude

$$
\partial_{t}\left\langle E_{\mathrm{eff}}\right\rangle_{\mathrm{fb}}=-\left(\partial_{t} V_{\max }\right)\left\langle\cos ^{2}(\tilde{k} X)\right\rangle .
$$

It is clear from this expression that the "bang-bang" feedback strategy from the simple cooling algorithm, where the potential amplitude is switched cyclically and suddenly between two values, maximizes the energy extraction rate if we consider only algorithms with a cyclic modulation of the potential within that range. In fact, we should switch the potential low when the value of $-\left\langle\cos ^{2}(\tilde{k} X)\right\rangle$ is maximized (i.e., the modulus is minimized), thus maximizing the energy extracted from the atom. Then we should switch the potential high when $-\left\langle\cos ^{2}(\tilde{k} X)\right\rangle$ is minimized, thus minimizing the energy transferred to the atom when completing the modulation cycle.

When we must rely on the Gaussian estimator to determine when to switch the potential, we switch the potential low or high when the estimated quantity

$$
y_{\text {est }}:=-\exp \left(-2 \widetilde{k}^{2} V_{X}^{\mathrm{e}}\right) \cos \left(2 \tilde{k}\left\langle\langle X\rangle_{\mathrm{e}}\right\rangle\right)
$$


is maximized or minimized, respectively. Doing so involves an additional technical complication, however, since at any given instant, we must predict whether or not $y_{\text {est }}$ is an extremal value. To do this we dynamically fit a quadratic curve to a history of these values (typically the last several hundred), and then trigger the feedback transitions on the slope of the fitted curve. This procedure is a convenient method for predicting the times when $y_{\text {est }}$ is maximized or minimized, and helps to reject the residual noise in the time evolution of this quantity. More formally, given a set of estimates $y_{\text {est }, n}$ corresponding to times $t_{n}$, we implement the curve fit of the function $a_{0}+a_{1} x+a_{2} x^{2}$ to the last $q$ values of $y_{\text {est, } i}$. We will defer the discussion of the computational efficiency of this algorithm until Sec. IIIC2.

It may seem strange that this algorithm makes use of past state information, when all the necessary information should in principle be contained in the current estimate of the state. Indeed, it is clear from the quantum Bellman equation $[8,11,13]$ that the optimal control algorithm is given by only looking forward in time given the current state. In practice, such an algorithm is difficult to realize, and the present algorithm is likely suboptimal but robust and effective.

We also note that since we are triggering in this algorithm on the estimated value of $-\left\langle\cos ^{2}(\widetilde{k} X)\right\rangle$, it seems that it could be much simpler to simply trigger on the detector photocurrent $d \widetilde{r}(t)$, which from Eq. (13) we know is a direct measure of this quantity plus noise. In fact, we can use the same curve-fitting procedure on $d \widetilde{r}(t)$ to reject the noise on the signal. However, as we will see, this procedure does not work nearly as well as with the Gaussian estimator, because the signal $d \widetilde{r}(t)$ is noise dominated, and the Gaussian estimator acts as a nearly optimal filter for the useful information from this signal, in the same sense as a Kalman filter $[12,58,68]$.

\section{Cooling limits}

At this point, we can work out a simple theory of cooling for the improved cooling algorithm developed in the previous section. When we switch the optical potential low, the change in energy from Eq. (22) is given by

$$
\Delta\left\langle E_{\mathrm{eff}}\right\rangle_{\mathrm{fb}}=-4 \varepsilon V_{\max }\left\langle\cos ^{2}(\tilde{k} X)\right\rangle,
$$

where we have taken $\varepsilon_{1}=\varepsilon_{2}=\varepsilon$, so that the potential amplitude is switched from $(1+\varepsilon)^{2} V_{\max }$ to $(1-\varepsilon)^{2} V_{\max }$. When we switch high, the expression for the energy change is the same except for an overall minus sign.

Now we consider these expectation values for a particle very close to the ground state, and we will first consider the case where all the excess motional energy is associated with the motion of the wave-packet centroid. For the ground state itself, an equal part $E_{0} / 2$ of the ground-state energy $E_{0}$ is associated with each of the kinetic and potential parts of the effective Hamiltonian. If we define $\Delta E:=\left\langle E_{\text {eff }}\right\rangle-E_{0}$ as the small, additional motional energy above the ground state, then as the displaced wave packet (coherent state) evolves in the nearly harmonic potential, the value $V_{\max }\langle[1$ $\left.\left.-\cos ^{2}(\tilde{k} X)\right]\right\rangle$ of the potential part of the atomic energy oscil- lates between $E_{0} / 2$ and $E_{0} / 2+\Delta E$. Then the best cooling that can be achieved for this state corresponds to two up and two down transitions per oscillation period (which is unity in our scaled units). Inserting these extremal values of $-V_{\max }\left\langle\cos ^{2}(\widetilde{k} X)\right\rangle$ into Eq. (24) and the corresponding expression for upward transitions gives the cooling rate of

$$
\Delta\left\langle E_{\mathrm{eff}}\right\rangle_{\mathrm{fb}}=-8 \varepsilon\left(\left\langle E_{\mathrm{eff}}\right\rangle-E_{0}\right)
$$

per unit time.

In practice, the cooling algorithm switches more often than in this estimate due to noise on the signal. However, in such cases we might still expect that Eq. (25) is a reasonable estimate for the cooling rate. Even though the potential is switched more often, the switches are caused by noise and thus do not occur at optimal moments in time. Thus the amount of cooling per switching cycle is reduced, and we observe that the net cooling effect per unit time is near the optimal rate of Eq. (25), presumably because the cooling algorithm is not too sensitive to the noise. If we then assume that heating due to spontaneous emission is negligible compared to measurement heating, then the steady-state motional energy is obtained when the cooling rate in Eq. (25) balances the heating rate which we derive in Appendix B [Eq. (B7)]. This gives the steady-state energy

$$
\left\langle E_{\text {eff }}\right\rangle_{\mathrm{SS}}=\frac{E_{0}}{1-\beta},
$$

where $\beta:=\Gamma \widetilde{k}^{4} / 2 \varepsilon$.

The other extreme case we will consider is where the motional energy in excess of the ground-state energy is associated purely with squeezing of the wave packet and not with the centroid. In this case, $V_{\max }\left\langle\cos ^{2}(\widetilde{k} X)\right\rangle$ oscillates between the values $\left(\left\langle E_{\text {eff }}\right\rangle \pm \sqrt{\left\langle E_{\text {eff }}\right\rangle^{2}-E_{0}^{2}}\right) / 2$, giving the cooling rate of

$$
\Delta\left\langle E_{\mathrm{eff}}\right\rangle_{\mathrm{fb}}=-8 \varepsilon \sqrt{E_{\mathrm{eff}}^{2}-E_{0}^{2}}
$$

per unit time. Then the condition that this cooling rate balances the heating rate in Eq. (B7) implies

$$
\left\langle E_{\mathrm{eff}}\right\rangle_{\mathrm{SS}}=\frac{E_{0}}{\sqrt{1-\beta^{2}}}
$$

for the steady-state energy. But in the regime where these theories are valid (i.e., $\left\langle E_{\text {eff }}\right\rangle_{S S}-E_{0} \ll E_{0}$ or $\beta \ll 1$ ), the centroid result of Eq. (26) is always larger than the squeezing result of Eq. (28). Thus, we expect that the centroid limit [Eq. (26)] is the more appropriate cooling limit.

Equation (26) predicts that the atomic motion will cool essentially to the ground state so long as $\beta \leq 1 / 2$. Thus, as various parameters are changed, there are "border" values that divide the parameter space according to whether or not the atomic motion is cooled to the ground state. For example, the atom will cool to nearly the ground state so long as the "bang amplitude" $\varepsilon$ is larger than the border value

$$
\varepsilon_{\mathrm{b}}=\Gamma \tilde{k}^{4} .
$$


Otherwise, the steady-state energy should be substantially higher, and the atomic motion may not even cool at all. However, this simple theory is only valid at small energies, so such behavior should not necessarily be predicted well by this theory. Additionally, this theory does not account for how well the Gaussian estimator tracks the true atomic state, and thus we would expect the actual steady-state temperatures to be higher than predicted by Eq. (26).

One final modification to this cooling theory is necessary, since as we discuss below, parity considerations show that half of the atoms can cool at best to the first excited band of the optical potential. To take this effect into account, we simply modify Eq. (26) by replacing $E_{0}$ by the average of the ground and first excited band energies $\left(E_{0}+E_{1}\right) / 2$. But in the harmonic approximation, $E_{0}=\pi$ and $E_{1}=3 \pi$, so the cooling limit becomes

$$
\left\langle E_{\text {eff }}\right\rangle_{\mathrm{SS}}=\frac{2 \pi}{1-\beta},
$$

This modification is simple because the centroid argument above only assumed that the atomic state is near the band with the lowest achievable energy. The odd-parity atoms have the first excited state as their lowest achievable state, and the above argument applies just as well to states near this effective ground state. Thus, the ensemble average amounts to simply averaging over the two possible steady-state energies.

\section{SIMULATIONS: ADIABATIC APPROXIMATION}

\section{A. Stochastic Schrödinger equation}

Simulating the simplified dynamics in the adiabatic approximation according to the SME in Eq. (14) is much less numerically intensive than simulating the full SME given in Eq. (7). Because of this, we will perform an extensive analysis of the behavior of the feedback control in this section using simulations in the adiabatic approximation. We will then verify that these simulations indeed provide a good description of the dynamics by performing a limited number of simulations of the full SME in the following section.

To perform our simulations, we first note that we may view any SME as being generated by averaging a stochastic Schrödinger equation (SSE), where the average is taken over all the signals that the observer fails to measure $[54,69]$. The unobserved signals in our case are the spontaneous emission from the atom and the part of the cavity output that is not measured due to photodetector inefficiency $(\eta<1)$. We therefore replace nature with a formal omniscient observer, unknown to the observer who will effect control. The omniscient observer measures everything that the control observer does not, and also has access to the control observer's measurement record. From the omniscient observer's point of view, the evolution of the system is described by an SSE. We can simulate the system by integrating this SSE, and even though it does not provide us with the control observer's state of knowledge, which would require averaging over all possible measurement results of the omniscient observer, it does generate instances of the control observer's appropriate measurement record. The control observer can then use this measurement record to obtain an estimate of the state of the system by integrating the Gaussian estimator derived above [13]. Since the "correct" state estimate, given by integrating the full SME, is not required, we can obtain a full simulation of the feedback control process by integrating the SSE, which requires far fewer resources than integrating the SME.

The normalized Schrödinger equation corresponding to the master equation (14) that we integrate numerically is

$$
\begin{aligned}
d|\psi\rangle= & -i H_{\mathrm{eff}}|\psi\rangle d t-\frac{\Gamma}{4} \cos ^{2}(2 \widetilde{k} X)|\psi\rangle d t \\
& +\frac{\Gamma}{2}\langle\cos (2 \widetilde{k} X)\rangle \cos (2 \widetilde{k} X)|\psi\rangle d t+\frac{\Gamma}{4}\langle\cos (2 \widetilde{k} X)\rangle^{2}|\psi\rangle d t \\
& +\sqrt{\frac{\Gamma}{2}}[\langle\cos (2 \widetilde{k} X)\rangle-\cos (2 \widetilde{k} X)]|\psi\rangle d W,
\end{aligned}
$$

which generates trajectories with the same measure as the SME. Since we are integrating the SSE in lieu of the SME, there are a few subtleties related to the measurement noise to be accounted for. In particular, the Wiener increment $d W$ in the SSE (31) is not equivalent to the Wiener increment in expression (13) for the measurement record if $\eta<1$, because the measured noise does not fully represent the "true" noise. Rather, the quantity $\sqrt{\eta} d W$ in the photocurrent expression should be replaced by a measured noise $d W_{\eta}$, given in terms of the full noise of the SSE by

$$
d W_{\eta}=\eta d W+\sqrt{\eta(1-\eta)} d W_{\text {aux }},
$$

where $d W_{\text {aux }}$ is an auxiliary Wiener increment, so that $d W_{\eta}^{2}$ $=\eta d t$, and a fraction

$$
d W_{\text {unm }}=(1-\eta) d W-\sqrt{\eta(1-\eta)} d W_{\text {aux }}
$$

of the true noise is not measured by the control observer (i.e., $\left.d W_{\eta}+d W_{\mathrm{unm}}=d W\right)$. The photocurrent in Eq. (13) then becomes

$$
d \widetilde{r}(t)=-\sqrt{8 \eta^{2} \Gamma}\left\langle\cos ^{2}(\widetilde{k} X)\right\rangle d t+d W_{\eta},
$$

and the estimated Wiener increment is given in terms of the measured noise as

$$
\begin{aligned}
d W_{\mathrm{e}}= & \frac{d W_{\eta}}{\sqrt{\eta}}+\sqrt{2 \eta \Gamma}\left[\exp \left(-2 \widetilde{k}^{2} V_{X}^{\mathrm{e}}\right) \cos \left(2 \widetilde{k}\langle X\rangle_{\mathrm{e}}\right)\right. \\
& -\langle\cos (2 \tilde{k} X)\rangle] d t
\end{aligned}
$$

which still reflects the difference between the actual and estimated quantum states in the same way as before.

\section{B. Details of the simulations}

For the simulations, we choose a "canonical" set of experimentally realistic parameters that will put us in the regime where adiabatic elimination of the cavity and internal atomic states should be valid. Using the cesium $\mathrm{D}_{2}$ line as the atomic transition, we have $m=2.21 \times 10^{-25} \mathrm{~kg}$ and $\omega_{0} / 2 \pi=351.7 \mathrm{THz}$; we assume that the cavity subtends a small solid angle and thus that the spontaneous emission rate 
is given approximately by the free-space value of $\gamma / 2 \pi$ $=5.2 \mathrm{MHz}$. For the cavity parameters, we choose values similar to those used in recent CQED experiments $[3,5,70]$, yielding an energy decay rate of $\kappa / 2 \pi=40 \mathrm{MHz}$, an atomfield coupling constant of $g / 2 \pi=120 \mathrm{MHz}$, and a mean intracavity photon number of $\alpha=1$. However, we will consider a much larger detuning of $\Delta / 2 \pi=4 \mathrm{GHz}$ to the red of the atomic resonance than was used in the experiments, in order to work in a dispersive regime where the adiabatic approximation should work well. The scaled parameters corresponding to these physical values are $\Gamma=23.6$ and $\tilde{k}=0.155$. The scaled depth of the optical potential is $V_{\max }=\pi / \widetilde{k}^{2}=131$, which corresponds to an atomic speed of $14.7 \mathrm{~cm} \mathrm{~s}^{-1}$ and is of sufficient depth that the lowest two band energies $E_{0}$ $=3.12$ and $E_{1}=9.33$ are close to the corresponding values in the harmonic approximation of $\pi$ and $3 \pi$, respectively (with 27 trapped bands in the optical potential and a ground-band width of $1.7 \times 10^{-12}$, so that tunneling transitions between wells are highly suppressed). Also, for the canonical parameter set we choose a feedback amplitude $\varepsilon=0.1$ (easily accomplished experimentally) and the idealized detection efficiency of $\eta=1$. The goal of our analysis will be to understand the dynamics of the system under these ideal conditions and then to understand how variations in these parameters influence the dynamics.

The optical potential in the simulations spanned 24 wells, corresponding to the cavity lengths in the Caltech experiments [70]. To crudely model the sticking behavior of an atom when colliding with a mirror, we implemented absorbing boundary conditions, where the absorption ramped smoothly on in the last half of each of the boundary potential wells. We explicitly conditioned the simulations on the fact that they were not lost, mimicking the experimental situation where a run with a lost atom would simply be repeated. However, given the fact that the cavity is relatively long compared to the optical potential period, these details do not substantially influence the results of the calculation.

To understand the typical dynamics of this problem, we consider ensemble averages. We consider the experimental situation where atoms dropped one at a time have substantial motional energy after loading into the optical potential, but only atoms that are well trapped are candidates for further cooling. Thus, we assume that all atoms in the simulation ensemble have a centroid energy of 84.2 , which corresponds to a stationary atom at a distance of 6 away from the bottom of a well (where the well period is $\pi / \tilde{k}=20.3$ ), and we distribute the initial locations of the atoms uniformly between the bottom of the well and this maximum distance. The atoms in the simulation begin in a coherent state with $V_{x}=V_{p}$ $=1 / 2$ in the center well of the cavity. We do not expect that the steady-state results are sensitive to the details of these initial conditions. Except where noted otherwise we plot averages over 128 trajectories; in plots where we inspect the variation of ensemble averages as a function of a parameter, each ensemble average is computed with respect to an independent set of random-number seeds.

We obtained numerical solutions to the SSE (31) using the order 1.5 (strong), implicit, stochastic Runge-Kutta (SRK) algorithm in Ref. [71] as part of an operator-splitting method. To evolve the system over a large step of $\Delta t$ $=0.0005$, we first used the SRK evolver to evolve the wave function according to the SSE without the kinetic $\pi P^{2}$ term in substeps of size $\Delta t / 8$ over an interval $\Delta t / 2$. Next, we applied the operator $\exp \left(-i \pi P^{2} \Delta t\right)$ after a Fourier transform to momentum space. Finally, after transforming back, we again evolved according to the SRK algorithm by another interval $\Delta t / 2$ (in four steps) to complete the full $\Delta t$ step. This operator splitting preserves the order 1.5 (temporal) convergence of the SRK approximation. The spatial grid contained 2048 points. The space and time discretizations were found to give adequate convergence when computed in 32-bit precision for most of the trajectories, although a minority (typically only a few percent) displayed some sensitivity to these numerical parameters due to their proximity to unstable hyperbolic points in phase space. However, for the cooling simulations, the steady-state energies were well converged and not affected by this sensitivity, since the atoms only saw the harmonic portions of the potential wells.

The Gaussian estimator begins in each case with the impure-state initial conditions $\langle X\rangle_{\mathrm{e}}=6,\langle P\rangle_{\mathrm{e}}=0, \quad V_{X}^{\mathrm{e}}=V_{P}^{\mathrm{e}}$ $=1 / \sqrt{2}$, and $C_{\mathrm{e}}=0$. As we argued above in Sec. III A, this choice of the initial estimate is arbitrary but sufficient for our purposes, and we will see in the next section that the cooling performance is insensitive to any "reasonable" initial estimate. The estimator is updated with a time step of $\Delta t_{\mathrm{G}}=\Delta t$ using the simple Euler method [71] (both to keep the calculations simple and because, as we discussed in Sec. III A, $d W_{\mathrm{e}}$ is not the usual Wiener increment and thus violates assumptions used in constructing higher-order integrators). Some extra measures are needed to guard against instabilities in the estimator evolution, which are due to two possible reasons. First, the low-order numerical integration of the stochastic estimator equations with a relatively large time step tends to be unstable, especially when the occasional large fluctuation occurs. Second, while Gaussian approximations to Hamiltonian evolution (or even evolution under an unconditioned Lindblad master equation) can be thought of as resulting from a variational principle, and hence inherently stable, the Gaussian approximation to the conditioned SME cannot, and thus does not inherit these strong stability properties. We explicitly detect the four unphysical conditions: negative position variance $\left(V_{X}^{\mathrm{e}}<0\right)$, negative momentum variance $\left(V_{P}^{\mathrm{e}}<0\right)$, phase-space area below the Heisenberg limit $\left(A_{\mathrm{e}}:=\left[V_{X}^{\mathrm{e}} V_{P}^{\mathrm{e}}-C_{\mathrm{e}}^{2}\right]^{1 / 2}<1 / 2\right)$, and large position variance $\left(V_{X}^{\mathrm{e}} \gg 1\right.$, indicating the atom is not localized within the cavity). If any of these conditions are detected, the variances are reset according to the initial values $V_{X}^{\mathrm{e}}=V_{P}^{\mathrm{e}}=1 / \sqrt{2}, C_{\mathrm{e}}=0$, while the means $\langle X\rangle_{\mathrm{e}}$ and $\langle P\rangle_{\mathrm{e}}$ are not modified; the measurement information will quickly restore the correct estimator values. For a typical cooling simulation with the canonical parameters, the estimator must be reset in about $95 \%$ of the trajectories, with trajectories on average requiring three resets due to the area condition (the other conditions producing negligible numbers of resets in comparison). Also, to help alleviate the number of resets caused by this condition, we instead implement the condition that resets only occur when $A_{\mathrm{e}}<1 / 4$. This situation may seem curious, as the Gaussian area evolves according to 

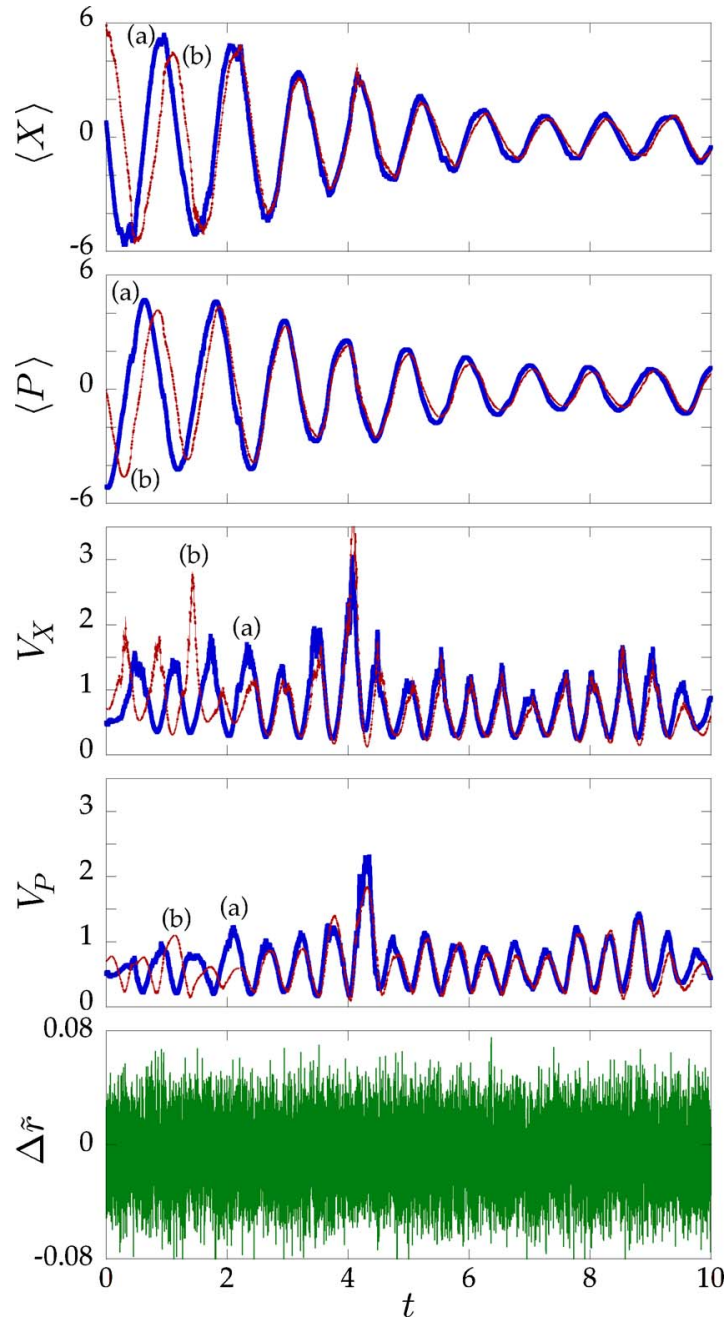

FIG. 1. (Color online) Example of locking behavior of the Gaussian estimator for centroids and variances. The heavy lines (a) are the expectation values for the "true" wave packet, while the light lines (b) are the corresponding Gaussian estimator quantities. The improved feedback cooling algorithm was switched on at $t=2$. The bottom graph shows the corresponding photodetector signal increments $\Delta \widetilde{r}$, plotted for the time increments of $\Delta t_{\mathrm{G}}=0.0005$ used by the estimator.

$$
\begin{aligned}
d\left(A_{\mathrm{e}}^{2}\right)= & \Gamma \widetilde{k}^{2} V_{X}^{\mathrm{e}}\left[1-\exp \left(-8 \widetilde{k}^{2} V_{X}^{\mathrm{e}}\right) \cos \left(4 \widetilde{k}\langle X\rangle_{\mathrm{e}}\right)\right] d t \\
& -8 \eta \Gamma \widetilde{k}^{2} V_{X}^{\mathrm{e}} A_{\mathrm{e}} \exp \left(-4 \widetilde{k}^{2} V_{X}^{\mathrm{e}}\right) \sin ^{2}\left(2 \widetilde{k}\langle X\rangle_{\mathrm{e}}\right) d t \\
& -8 \eta \Gamma \widetilde{k}^{4} V_{X}^{\mathrm{e} 2}\left[1-4 \widetilde{k}^{2} V_{X}^{\mathrm{e}} \exp \left(-2 \widetilde{k}^{2} V_{X}^{\mathrm{e}}\right)\right] \\
& \times \exp \left(-2 \widetilde{k}^{2} V_{X}^{\mathrm{e}}\right) \cos ^{2}\left(2 \widetilde{k}\langle X\rangle_{\mathrm{e}}\right) d t-\sqrt{2 \eta \Gamma} \widetilde{k}^{2} V_{X}^{\mathrm{e}} \\
& \times\left[1-4\left(\widetilde{k}^{2} V_{X}^{\mathrm{e}}+A_{\mathrm{e}}\right) \exp \left(-2 \widetilde{k}^{2} V_{X}^{\mathrm{e}}\right)\right] \\
& \times \cos ^{2}\left(2 \widetilde{k}(X\rangle_{\mathrm{e}}\right) d W_{\mathrm{e}},
\end{aligned}
$$

whence it follows after some examination that $A_{\mathrm{e}} \geqslant 1 / 2$ for all time if this is true initially. Thus, these resets are numerical artifacts of evolving the estimator using a finite time step and a low-order numerical method; in the simulations, reducing the time step $\Delta t_{\mathrm{G}}$ by a factor of 10 reduces the average number of resets due to the area condition to 1.1 (with 0.8 resets on average due to the $V_{X}^{\mathrm{e}}<0$ condition). However, we continue to use the larger step size, which is more reasonable to implement in practice given technological constraints on the speed of calculations for the forseeable future. For somewhat smaller measurement efficiencies, the estimator requires fewer resets (e.g., about $12 \%$ of the trajectories for $\eta=0.8$, and only 1.7 resets on average per trajectory), since the steady-state area is larger (corresponding to a state with lower purity, as $\operatorname{Tr}\left[\rho_{\mathrm{e}}^{2}\right]=1 /\left(2 A_{\mathrm{e}}\right)[72]$, where $\rho_{\mathrm{e}}$ is the estimated density operator).

Because the estimator locks on quickly with the canonical parameters, the feedback cooling algorithm is turned on at time $t=2$. For the improved cooling algorithm, we find that fitting to the last 300 observations (spaced apart in time by $\left.\Delta t_{\mathrm{G}}\right)$ is optimal.

\section{Estimation and cooling dynamics}

Now we will evaluate the performance of the two main components of the quantum feedback cooling system, the estimator and the cooling algorithm. Henceforth, we will only study the performance of the improved cooling algorithm, except where noted otherwise. Figure 1 shows a typical trajectory undergoing cooling and compares some of the "true" moments computed from the atomic wave function (i.e., those computed with respect to the result of integrating the SME of the omniscient observer) with the moments from the estimator. The centroids and variances of the estimator lock on by the time that the cooling algorithm is switched on $(t=2)$, and the estimator provides a reasonably faithful representation of the atomic dynamics. It is especially important that the variances track the atomic wave packet accurately in order to make the improved cooling work effectively. Figure 1 also shows the measurement record increments

$$
\Delta \widetilde{r}(t):=\int_{t}^{t+\Delta t_{\mathrm{G}}} d \widetilde{r}(t)
$$

corresponding to the time increments $\Delta t_{\mathrm{G}}=0.0005$ used in the Gaussian-estimator integration. The measurement record appears to be noise dominated, but nevertheless contains information about the quantum state in the time-dependent offset level of the noise. The action of the estimator is such that it "demultiplexes" the information about the motions of the centroids and variances, even though they are encoded in the same frequency range in the photocurrent signal. One can get a sense for how this works from the form of Eqs. (19). For example, the measurement $\left(d W_{\mathrm{e}}\right)$ terms in the $d\langle X\rangle_{\mathrm{e}}$ and $d V_{X}^{\mathrm{e}}$ equations are weighted by $\sin \left(2 \widetilde{k}\langle X\rangle_{\mathrm{e}}\right)$ and $\cos \left(2 \widetilde{k}\langle X\rangle_{\mathrm{e}}\right)$, respectively. Thus, the incoming measurement information influences $\langle X\rangle_{\mathrm{e}}$ or $V_{X}^{\mathrm{e}}$, depending on the state of the estimator: if the estimator is centered in a well, variations in $\left\langle\cos ^{2} \tilde{k} X\right\rangle$ (i.e., the photocurrent) are presumed to be caused by variations in $V_{x}$, while such variations are attributed to $\langle X\rangle$ if the estimator is centered on the side of a well. The estimator can also gain information from the absolute value of the measurement record-as we discussed in Sec. III C, energy in only the variance degree of freedom can produce transient 


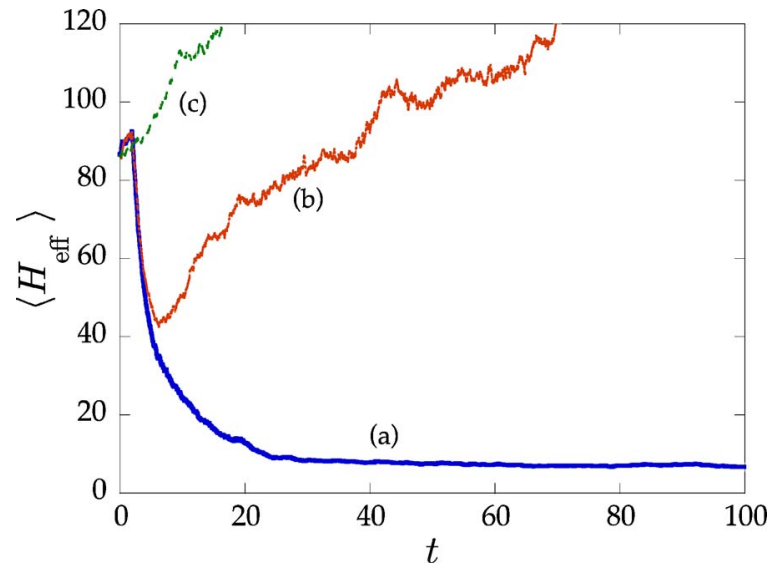

FIG. 2. (Color online) Evolution of the mean energy $\left\langle H_{\text {eff }}\right\rangle$ (relative to the minimum potential energy), comparing the performance of different cooling algorithms. Heavy solid line (a): improved cooling algorithm. Solid line (b): centroid-only cooling algorithm. Dashed line (c): no cooling algorithm. Each line is an average of 128 trajectories; parameters are for the canonical set.

values of $V_{\max }\left\langle\left[1-\cos ^{2}(\tilde{k} X)\right]\right\rangle$ below $E_{0} / 2$, whereas energy in only the centroid cannot. This subtle unraveling of the measurement information into the estimated state is automatic in the measurement formulation we have used here.

Figure 2 compares the ensemble-averaged evolution of the energy $\left\langle E_{\text {eff }}\right\rangle$ in the case where the atoms are not cooled to the cases where the atoms are cooled by the centroid-only and improved cooling algorithms. When the atoms are not actively cooled, the energy simply increases linearly in time. The initial heating rate of $\partial_{t}\left\langle E_{\text {eff }}\right\rangle=1.78$ predicted by Eq. (B6) for measurement backaction is substantially smaller than the initial simulated heating rate of 2.1(1). We can understand this discrepancy, since the initial conditions in the simulation are uniformly distributed in phase space along a constant-energy surface, weighting more heavily the gradients of the potential where heating is maximized; Eq. (B6) underestimates the heating rate by assuming a uniform distribution in configuration space.

The simple, centroid-based cooling algorithm cools the atoms rapidly for short times, but around $t=10$, the cooling stops and the atoms begin to heat back up, apparently without bound. Clearly, we expect some heating from the squeezing effects discussed above. Furthermore, we expect that when the centroid cools and the variance becomes much larger than the mean-square amplitude of centroid motion, the centroid evolution will be dominated by measurement (projection) noise, and thus the feedback signal will also be determined mostly by random noise from the measurement. In this state, the cooling algorithm is ineffective and leads to heating. This process runs away, since there is no mechanism to cool the energy associated with the variances (squeezing).

On the other hand, the improved cooling algorithm also rapidly cools the atoms and the atomic energies settle to a well-defined steady state, which already demonstrates the potential utility of this cooling algorithm for long-term storage of the atoms. For the moment, we will defer the question of how closely the atoms are cooling to the lowest energy

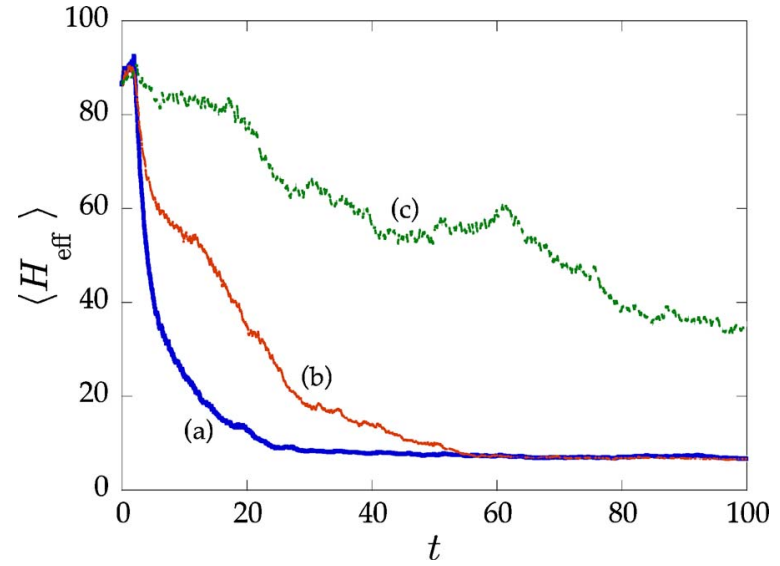

FIG. 3. (Color online) Evolution of the mean energy $\left\langle H_{\text {eff }}\right\rangle$, comparing the performance of the improved cooling algorithm with different initial (Gaussian) state estimates. Heavy solid line (a): estimate matches the canonical parameter set. Solid line (b): initial estimate is much more impure and substantially overlaps $(x, p)$ $=(0,0)$. Dashed line $(\mathrm{c})$ : same as the solid line, but centered on $(x, p)=(0,0)$.

band of the optical potential until we take a closer look at the atomic dynamics in the next section.

The cooling behavior that we see is also insensitive to the exact choice of the initial (Gaussian) state estimate, so long as we have made a reasonable choice. To illustrate this statement, Fig. 3 shows the ensemble-averaged cooling dynamics for two initial state estimates in addition to the canonical estimate that we use for the rest of the simulations. In the first modified initial estimate, we have selected $\langle X\rangle_{\mathrm{e}}=0.1$, $\langle P\rangle_{\mathrm{e}}=0, V_{X}^{\mathrm{e}}=1, V_{P}^{\mathrm{e}}=1$, and $C_{\mathrm{e}}=0$ for a much larger uncertainty product (lower purity $\operatorname{Tr}\left[\rho^{2}\right]$ ) and a substantial overlap with the other side of the potential well. We see that although the short-time cooling performance is slightly worse, the long-time cooling performance is the same as for the original initial estimator. This implies that the tracking behavior is initially worse for this initial estimator, but at sufficiently long times the estimator "forgets" its initial state and does a better job of tracking. The other initial estimator is the same as the previous case, but with $\langle X\rangle_{\mathrm{e}}=0$, so that the estimator is centered on the origin in phase space. Examination of the estimator equations (19) reveals that in this case the centroids $\langle X\rangle_{\mathrm{e}}$ and $\langle P\rangle_{\mathrm{e}}$ will remain zero for all time, effectively "freezing out" the estimator's centroid degree of freedom. The cooling behavior here is much worse than in the other two cases, demonstrating both that the estimator's variance degree of freedom is insufficient to mimic the dynamics of the true state and that it is important to exercise some caution when selecting an initial state estimate.

Finally, we return to the question raised in Sec. III B regarding the necessity of using the Gaussian estimator at all, in light of the fact that the measurement record itself provides a direct, albeit very noisy, measurement of the quantity $\left\langle\cos ^{2} \tilde{k} X\right\rangle$ used to determine the control switching times in the improved cooling algorithm. Figure 4 address this question by comparing the energy evolution under the improved cooling algorithm using the Gaussian estimator with the 


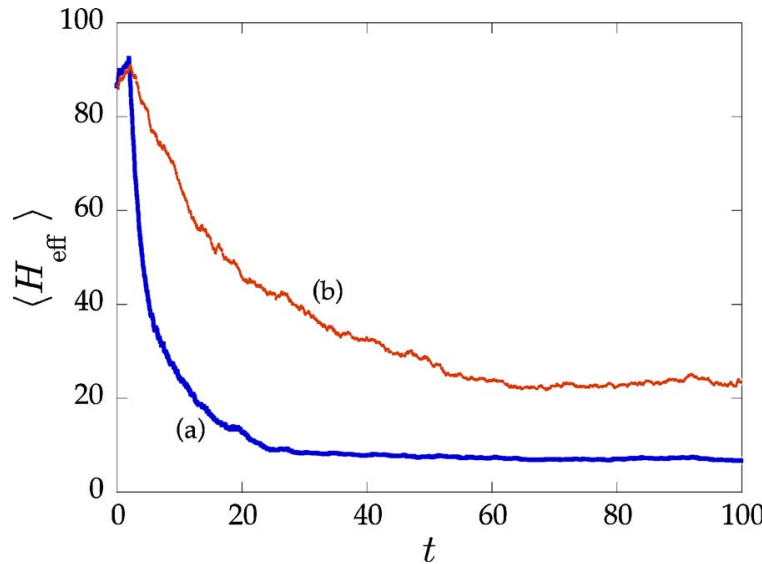

FIG. 4. (Color online) Evolution of the mean energy $\left\langle H_{\text {eff }}\right\rangle$, comparing the performance of the improved cooling algorithm [heavy solid line (a)] with that of cooling based directly on the homodyne signal [solid line (b)]. Heavy solid line: improved cooling algorithm. Each line is an average of 128 trajectories; parameters are for the canonical set.

same algorithm but based directly on the measurement record. In both cases, the quadratic curve was fitted on-thefly to the last 300 points in the measurement record to reduce noise effects. We found this number of points to be optimal for the canonical parameter set, and this optimal time interval over which the data are fit is tied directly to the time scales of the atomic motion. Although the direct-cooling case cools rapidly and settles to a lower temperature, it is clear that the cooling performance is greatly improved by the Gaussian estimator. The estimator acts as a nearly optimal noise filter, efficiently extracting the relevant information from the noisy measurement record.

\section{Parity dynamics}

We now consider the evolution of the parity of the conditioned atomic state due to the measurement. For this discussion, we will focus on the expectation values of the usual parity operator $\mathcal{P}$ [where $\mathcal{P} \psi(x)=\psi(-x)]$. For numerical purposes, though, we will instead use the reduced parity operator $\mathcal{P}^{\prime}:=\mathcal{R}^{\dagger} \mathcal{P} \mathcal{R}$, where the reduction operator $\mathcal{R}$ is defined by

$$
\mathcal{R} \psi(x):=\sum_{j=-\infty}^{\infty} \psi\left(x-\frac{j \pi}{\widetilde{k}}\right), \quad x \in\left[-\frac{\pi}{2 \widetilde{k}}, \frac{\pi}{2 \widetilde{k}}\right),
$$

which allows us to detect the parity of the atomic state with respect to any potential well in the cavity, rather than just the center well. We will simply use the notation " $\mathcal{P}$ " for both operators in the discussion, though, as the discussion holds for either operator. The initial conditions for the simulations, which correspond to Gaussian wave packets displaced in phase space from the origin in phase space, represent equal mixtures of odd and even parity $(\langle\mathcal{P}\rangle=0)$ to a good approximation. As time evolves, however, the parity of the conditioned atomic state evolves (in the adiabatic approximation) according to

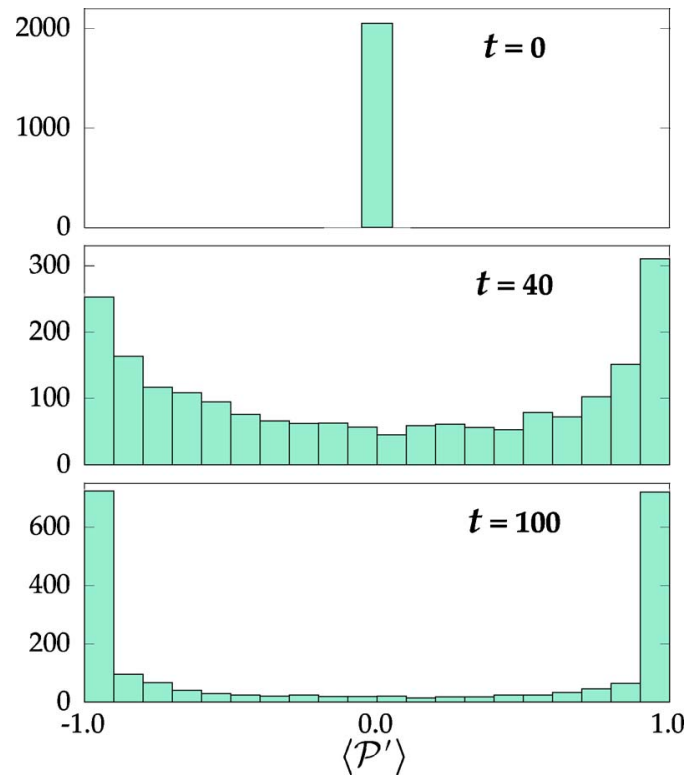

FIG. 5. (Color online) Histograms of the reduced parity $\left\langle\mathcal{P}^{\prime}\right\rangle$ for an ensemble of 2048 trajectories undergoing cooling, showing how parities evolve towards the extreme states of pure parity at late times.

$$
d\langle\mathcal{P}\rangle=-\sqrt{8 \eta \Gamma}[\langle\mathcal{P} \cos (2 \tilde{k} X)\rangle-\langle\mathcal{P}\rangle\langle\cos (2 \tilde{k} X)\rangle] d W .
$$

This effect is due only to the nonlinearity of the measurement term in the master equation (14); the parity under unconditioned evolution is invariant.

The reduced parity evolution for a simulated ensemble of trajectories undergoing measurement and cooling is shown in Fig. 5. It is clear that $\langle\mathcal{P}\rangle$ evolves towards the extreme values \pm 1 of parity for the simulated trajectories. This "parity purification" behavior is not immediately clear from the evolution equation (39), which states that $\langle\mathcal{P}\rangle$ evolves according to a diffusion process. The key point is that this diffusion process is nonstationary, since the diffusion rate depends on the parity, and indeed vanishes for states with pure parity. To see the final steady state directly, we consider the projectors $\mathcal{P}_{+}$ and $\mathcal{P}_{-}$for even and odd parity, respectively, defined by

$$
\mathcal{P}_{ \pm}:=\frac{1 \pm \mathcal{P}}{2}
$$

Then the product of the expectation values of these projectors evolves according to

$$
d\left[\left\langle\mathcal{P}_{+}\right\rangle\left\langle\mathcal{P}_{-}\right\rangle\right]=-8 \eta \Gamma\left(\left\langle\mathcal{P}_{+}\right\rangle\left\langle\mathcal{P}_{-}\right\rangle_{\wp}\right)^{2} d t,
$$

where the parity difference $\wp$ is given for states with $\left\langle\mathcal{P}_{ \pm}\right\rangle$ $\neq 0$ by

$$
\wp:=\frac{\left\langle\mathcal{P}_{+} \cos ^{2} \tilde{k} X\right\rangle}{\left\langle\mathcal{P}_{+}\right\rangle}-\frac{\left\langle\mathcal{P}_{-} \cos ^{2} \tilde{k} X\right\rangle}{\left\langle\mathcal{P}_{-}\right\rangle} .
$$

The magnitude of $\wp$ represents how well the measurement of $\cos ^{2} \tilde{k} X$ can resolve the parity of the atomic state, and it is 


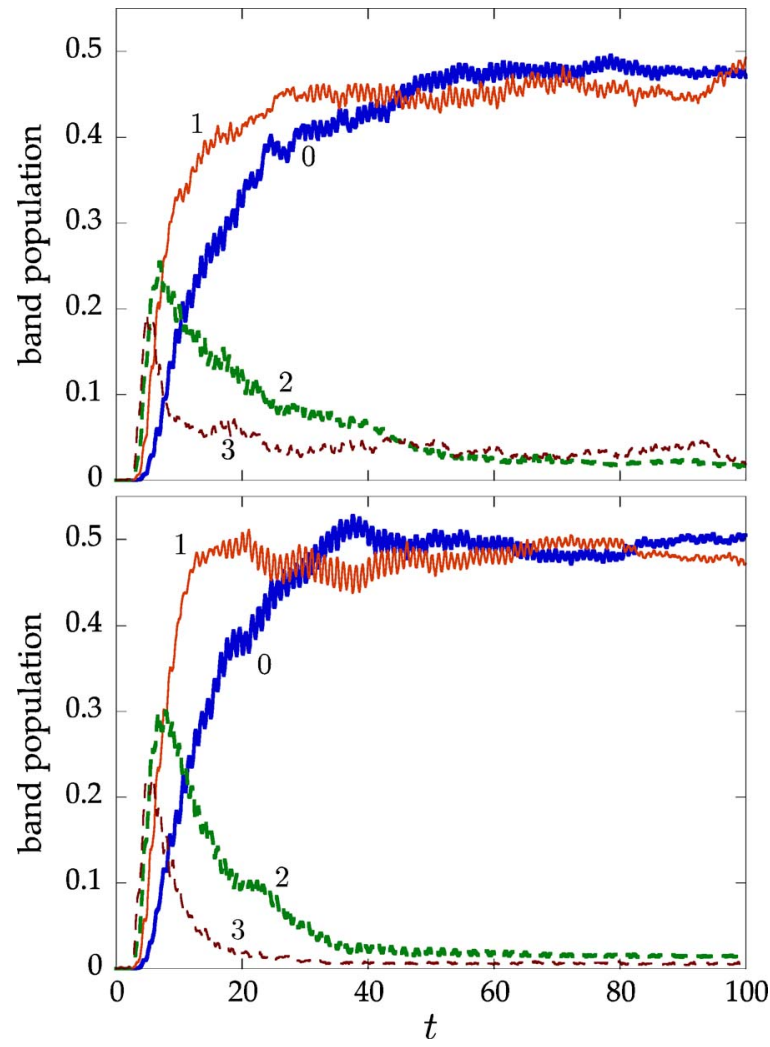

FIG. 6. (Color online) Evolution of the band populations for the lowest two (0 and 1) and next two (2 and 3$)$ energy bands of the optical lattice, averaged over 128 trajectories. Top: cooling based on the Gaussian estimator. Bottom: cooling based on perfect knowledge of the actual wave function.

nonzero for generic states. Thus, the quantity $\left\langle\mathcal{P}_{+}\right\rangle\left\langle\mathcal{P}_{-}\right\rangle$ damps monotonically to zero, which is only possible if the parity of the state becomes either pure even or pure odd. Given the initial conditions in the simulations, and the fact that the diffusion process in Eq. (39) is unbiased, we can expect that half of the trajectories will become even and half will become odd. As we mentioned above, this argument also applies to both the standard and reduced parity operators. However, the damping rate in Eq. (41) is greatly reduced for the standard parity operator when considering states localized in potential wells not centered on $X=0$.

This purification effect obviously has consequences for cooling the atomic motion. Even if the cooling algorithm can, in principle, cool the atomic motion faster than it is heated by the measurement, the cooling algorithm cannot affect the parity of the atomic state, and thus odd-parity states will not be cooled to the ground state of the optical potential. At best, then, the cooling algorithm can cool the atom to the ground energy band with probability $1 / 2$ and the first excited energy band with probability $1 / 2$. This cooling behavior is illustrated in Fig. 6, where the evolutions of the band populations are plotted for the two cases where cooling is based on the Gaussian estimator and directly on the true atomic wave function using the improved algorithm in both cases. We can clearly see that in steady state the lowest and first excited bands are about equally populated, with these bands accounting for $94 \%$ of the population in the Gaussian- estimator case and $98 \%$ of the population in the perfectknowledge case. Thus, this parity effect is the dominant limit to the atomic motional energy for the ensemble. However, this situation is essentially as good as cooling fully to the ground band, because the measurement can distinguish between the two possible outcomes (i.e., by resolving the two different possible steady-state potential energies). Experimentally, if the odd-parity outcome is detected, the atom can be transferred to the ground band by a coherent process (e.g., resonant modulation of the optical potential by an external field or two-photon, stimulated Raman transitions), or the state can simply be rejected, in hopes of obtaining the evenparity outcome on the next trial.

Finally, we comment on one odd aspect of the intermediate-time histogram $(t=40)$ in Fig. 5, which shows a marked asymmetry, even though the median value of $\langle\mathcal{P}\rangle$ is zero. Because we can write Eq. (39) in the form

$$
d\langle\mathcal{P}\rangle=-\sqrt{8 \eta \Gamma}\left\langle\mathcal{P}_{+}\right\rangle\left\langle\mathcal{P}_{-}\right\rangle_{\wp} d W,
$$

all of the asymmetry is due to the $\wp$ factor. Since this diffusion process mimics a quantum nondemolition measurement of the parity, we can interpret $\wp$ as an effective measurement strength that is larger for even-parity states than for odd states. This asymmetry is reasonable since the measurement and cooling processes produce even-parity states that are more localized than the odd ones. Thus, the even-parity states, being tightly localized at the field antinodes, produce more extreme values of the measurement record and are hence more easily distinguished from mixed-parity states. Again, however, this effectively asymmetric paritymeasurement process does not affect the long-time outcome that either parity will be selected with equal probability.

\section{E. Tests of cooling-limit theories}

Now we can examine the dependence of the steady-state cooling energies on the system parameters and test the validity of the cooling-limit theories in Sec. III C. Figure 7 shows the dependence of the simulated steady-state temperatures on the control-switching amplitude $\varepsilon$ in both the Gaussianestimator and perfect-knowledge cases. The simple cooling prediction of Eq. (30) is also shown here for comparison. The agreement between the theory and the perfectknowledge simulations is much better than between the theory and the Gaussian-estimator simulations, which is sensible because the simple theory does not attempt to account for the imperfections in how the estimator tracks the true state. While the (perfect-knowledge) steady-state energies consistently lie slightly below the theoretical prediction, the agreement is overall quite good, and the theory correctly predicts a sharp transition from efficient to inefficient cooling near the transition border $\varepsilon_{\mathrm{b}}=0.014$ (corresponding to the point $\beta \sim 1 / 2$ where the predicted steady-state energy is twice the smallest predicted value). Simply put, when the control transitions of the optical potential are sufficiently weak, the cooling algorithm does not cool the atoms at a rate sufficient to counteract the measurement heating (the system is no longer "controllable"), and so the atoms do not equilibrate at a very low temperature. The cooling and transition 


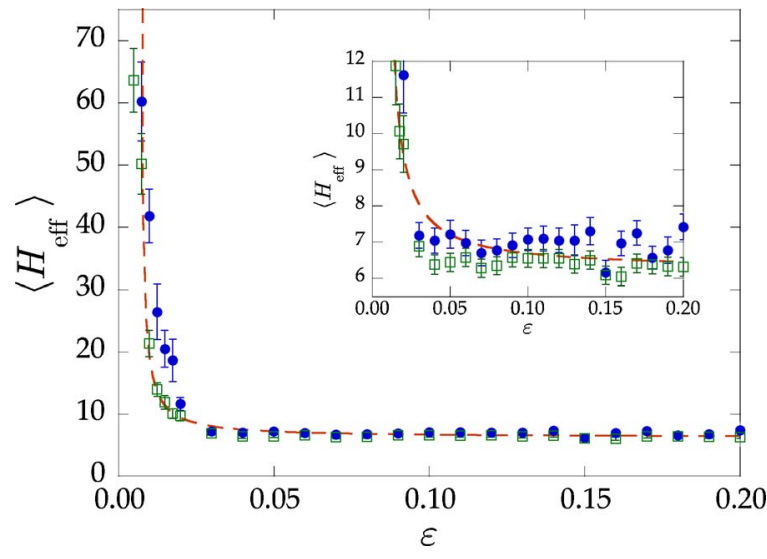

FIG. 7. (Color online) Final energies, measured over the interval from $t=90$ to 100 , as the switching amplitude $\varepsilon$ varies; other parameters match the canonical set. Circles: cooling based on the Gaussian estimator. Squares: cooling based on perfect knowledge of the actual wave function. Dashed line: simple cooling theory, Eq. (30). Inset: magnified view of the same data. Error bars reflect standard errors from averages over 128 trajectories.

behaviors are very similar in the Gaussian-estimator simulations, but these energies are overall slightly higher, again probably due to the inability of the Gaussian estimator to perfectly track the atomic state, even with $\eta=1$.

Similar behavior appears as the (unmodulated) optical potential depth $V_{\max }=\pi / \widetilde{k}^{2}$ varies, as shown in Fig. 8. For sufficiently large potential depth, the temperature is essentially constant, but if the potential depth is too weak, we again lose controllability and the cooling algorithm fails to be effective. There is a much more substantial difference between the Gaussian-estimator simulations and the simple cooling theory for small $V_{\max }$ compared to Fig. 7. This discrepancy is most likely due to the reduced confinement of the wave

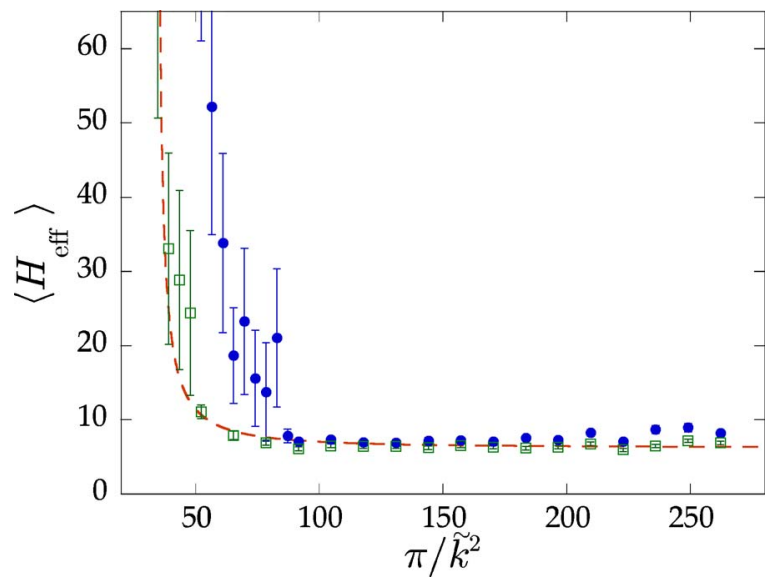

FIG. 8. (Color online) Final energies, measured over the interval from $t=90$ to 100 , as the unmodulated optical potential depth $V_{\max }=\pi / \tilde{k}^{2}$ varies; other parameters match the canonical set. Circles: cooling based on the Gaussian estimator. Squares: cooling based on perfect knowledge of the actual wave function. Dashed line: simple cooling theory, Eq. (30). Error bars reflect standard errors from averages over 128 trajectories.

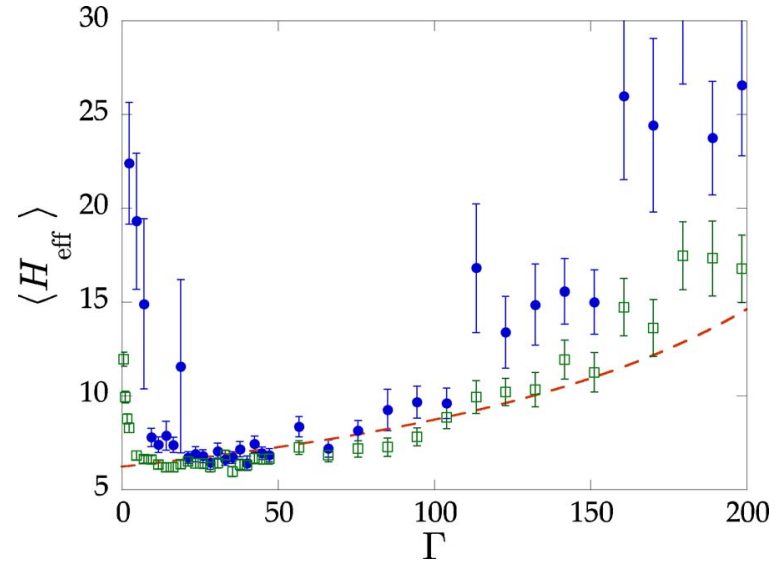

FIG. 9. (Color online) Final energies, measured over the interval from $t=90$ to 100 , as the effective measurement strength $\Gamma$ varies; other parameters match the canonical set. Circles: cooling based on the Gaussian estimator. Squares: cooling based on perfect knowledge of the actual wave function. Dashed line: simple cooling theory, Eq. (30). Error bars reflect standard errors from averages over 128 trajectories.

packet, which produces stronger anharmonic effects and thus a breakdown of the Gaussian approximation. The simulation data are still in good agreement with the simple theory.

The dependence of the steady-state energy on the measurement strength $\Gamma$ is shown in Fig. 9. In this case, the theory again matches the perfect-knowledge situation quite well. The cooling is best for moderately small values of $\Gamma$, and the simple theory predicts a transition to an uncooled state if $\Gamma$ exceeds the border value $\Gamma_{\mathrm{b}}=\varepsilon / \widetilde{k}^{4}=173$, since $\Gamma$ controls the rate of heating in the system. However, due to the functional form of Eq. (30), the "transition" here is much more gradual than the transition observed when varying $\varepsilon$. The Gaussian-estimator simulations again result in values that are consistently slightly above the perfect-knowledge energies. The difference is very pronounced for small $\Gamma$, where we might expect that the estimator is not gaining sufficient information from the measurement and cannot track the true state well enough to produce effective cooling (i.e., the system is no longer "observable"). The perfectknowledge energy data also show a slight upturn for small $\Gamma$ that is not predicted by the simple theory. This departure indicates the direct importance of the localization produced by the measurement in the cooling process: a localized phase-space distribution is responsible for larger fluctuations in $\left\langle\cos ^{2} \tilde{k} X\right\rangle$ than a delocalized distribution with the same energy, and from the discussion in Sec. III B, the cooling rate is directly proportional to the magnitude of these fluctuations. Thus, a sufficiently large value of $\Gamma$ must be chosen to maintain a localized atomic distribution and hence good cooling.

Thus far, we have focused on testing the theory of Eq. (30), which is the most relevant theory for a physical implementation of the cavity QED feedback system. However, we can also test the variance-only theory of Eq. (28) in the simulations, thereby giving indirect support to the more physi- 


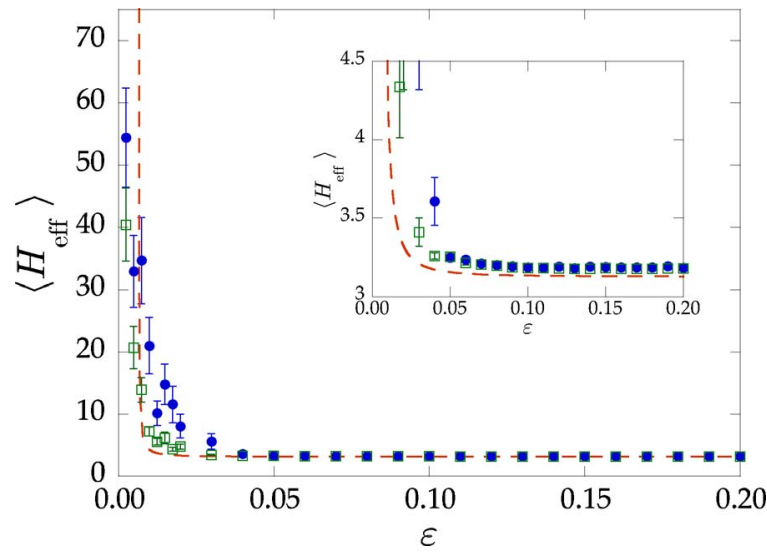

FIG. 10. (Color online) Final energies as in Fig. 7, but with initial position and momentum centroids (for both the atomic wave packet and Gaussian estimator) at zero. The dashed line now refers to the variance-dominated, even-parity theory of Eq. (28).

cally relevant theory. We can do this by changing the initial condition of the atomic wave packets to be a coherent (Gaussian) state centered on one of the potential wells with zero centroid momentum. From the form of the Gaussian estimator equations (28) we see that the wave-packet centroid will remain fixed at zero position and momentum, and thus all the energy will be associated with the wave-packet variances (in the harmonic approximation). The results of simulations of this type, where we also modify the initial condition of the Gaussian estimator to have the same centroid, are plotted in Fig. 10. The theory of Eq. (28) is also shown; note that we do not need to replace the $E_{0}$ in this expression with the average of $E_{0}$ and $E_{1}$, since the initial conditions have exactly even parity, which is then time invariant. The theory is again in good agreement with the perfect-knowledge data, both in the lower temperatures for large $\varepsilon$ and the sharper transition and lower transition border of $\varepsilon_{b}=0.0079$ (corresponding to $\beta \sim \sqrt{3} / 2$, again when the predicted energy is twice the minimum predicted value).

Finally, a note about the steady-state temperatures is in order for the cases where the Gaussian estimator is used for cooling. The ensemble-averaged, late-time energies in Figs. 7-10 for Gaussian-estimator cooling are slightly but consistently higher than the corresponding perfect-knowledge energies, suggesting that the cooling is somewhat less effective when the Gaussian estimator is used. However, these averages mask complicated dynamics that are illustrated in Fig. 11. Trajectories that have even parity at late times settle into a quiet equilibrium with nearly all the population in the ground energy band. On the other hand, trajectories that settle to odd parity have a more complicated equilibrium, where the atom is mostly in the first excited band but the evolution is punctuated by "bursts" of heating. This is an indication that in steady state, the Gaussian estimator is, unsurprisingly, a much better approximation for a wave packet close to the ground state than a wave packet close to the first excited state. As seen in Fig. 11, these heating episodes correspond to population being transferred to higher energy states of odd parity, a process distinct from the parity drift
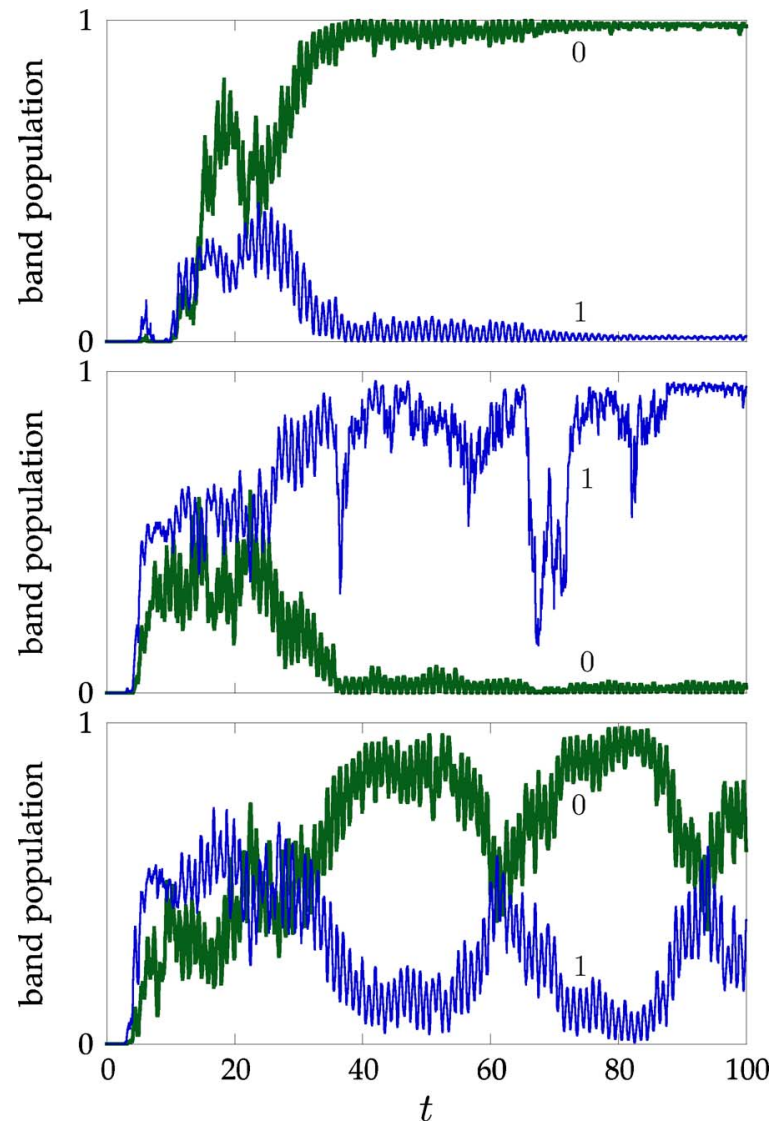

FIG. 11. (Color online) Evolution of the ground and first-excited band populations for three trajectories, illustrating typical behavior. Trajectories that purify to even parity (top) tend to settle to a quiet equilibrium in the ground state. Trajectories purifying to odd parity (middle) are characterized by dynamical equilibria, where periods in the first excited state are interrupted by episodes of higher energy. Trajectories that take longer to purify in parity (bottom) cool rapidly to the lowest two states, after which population is transferred between these two states as the parity diffuses. Parameters here correspond to the canonical set.

which, after the initial cooling, transfers population between the ground and first excited states. Again, if the goal of the cooling process is to prepare an atom in the ground state, the rate of success is even better than suggested by the ensemble-averaged energies, since cooling in the even-parity cases is essentially as good as if one had perfect knowledge of the actual wave function.

\section{SIMULATIONS: FULL ATOM-CAVITY DYNAMICS}

To verify the validity of the results of this paper, it is important to check the adiabatic approximation that leads to the reduced master equation (14). We thus carried out simulations of the full, coupled atom-cavity dynamics described by Eq. (7).

The simulation procedure is the same as before, except that we used the normalized Schrödinger equation corresponding to the master equation (7) 


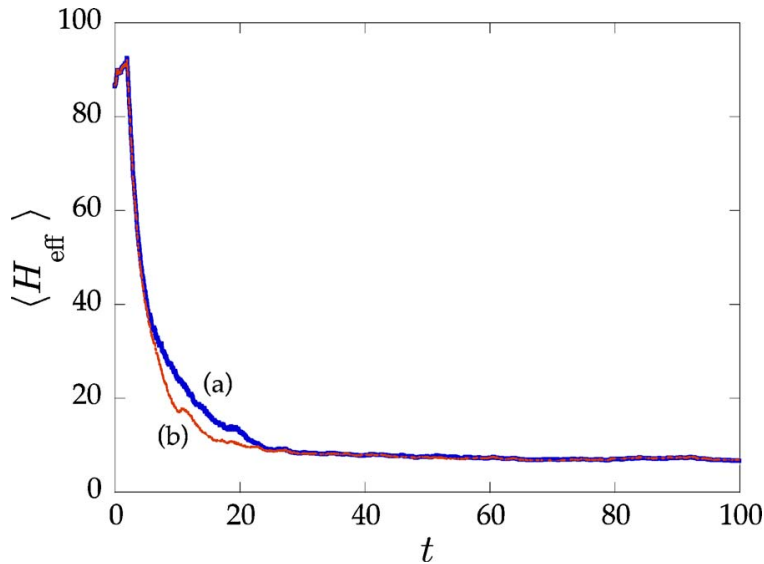

FIG. 12. (Color online) Ensemble energy evolution comparing the effect of the spatial grid size (cavity mirror separation) on the atomic evolution. Heavy solid line (a): canonical parameters (adiabatic approximation) spanning 24 optical potential wells. Light solid line (b): 6 potential wells. Each curve represents an average over 128 trajectories.

$$
\begin{aligned}
d|\psi\rangle= & -i H|\psi\rangle d t-\frac{\widetilde{\kappa}}{2} a^{\dagger} a|\psi\rangle d t+\frac{\widetilde{\kappa}}{2}\left\langle a+a^{\dagger}\right\rangle a|\psi\rangle d t \\
& -\frac{\widetilde{\kappa}}{8}\left\langle a+a^{\dagger}\right\rangle^{2}|\psi\rangle d t+\frac{\widetilde{\gamma}}{2}\left(\left\langle\sigma^{\dagger} \sigma\right\rangle-\sigma^{\dagger} \sigma\right)|\psi\rangle d t \\
& +\sqrt{\widetilde{\kappa}} a|\psi\rangle d W-\frac{\sqrt{\widetilde{\kappa}}}{2}\left\langle a+a^{\dagger}\right\rangle|\psi\rangle d W+\left(\frac{\sigma e^{i \tilde{u} X}}{\sqrt{\left\langle\sigma^{\dagger} \sigma\right\rangle}}-1\right) \\
& \times|\psi\rangle d N .
\end{aligned}
$$

We used the canonical parameter set described before. This set leads to scaled parameter values of $\tilde{\gamma}=190$ and $\widetilde{\kappa}=1460$ in addition to those used in the adiabatic-approximation simulations as described in Sec. IV B. The detuning $\Delta / 2 \pi$ $=4 \mathrm{GHz}$ from atomic resonance implies a wide separation of timescales in the problem. Thus we again evolved the system over a large step of $\Delta t=0.0005$ but in much smaller substeps of $\Delta t / 4096$, applying the kinetic evolution operator after half of the substeps. The spatial grid contained 512 points, spanning only 6 wells to make the computation more tractable. The impact of the smaller grid on the cooling was to slightly accelerate the cooling process, as illustrated in Fig. 12. This effect due to an effective evaporation of hot atoms, since we treat the mirrors as absorbing boundary condition as discussed above. We also used seven cavity states and two internal atomic states. Due to the small time step, we found it necessary to use 64-bit precision for these simulations.

The simulation results are shown in Fig. 13, where we see that there is only a small difference between the cooling performance in the adiabatic and full simulations. Also shown is a simulation with a detuning $\Delta / 2 \pi=2 \mathrm{GHz}$, evolved with substeps of $\Delta t / 2048$, to increase the nonadiabatic effects. The parameters were scaled to compensate. Even with this relatively close detuning, we see that the cooling dynamics are well described in the adiabatic approxima-

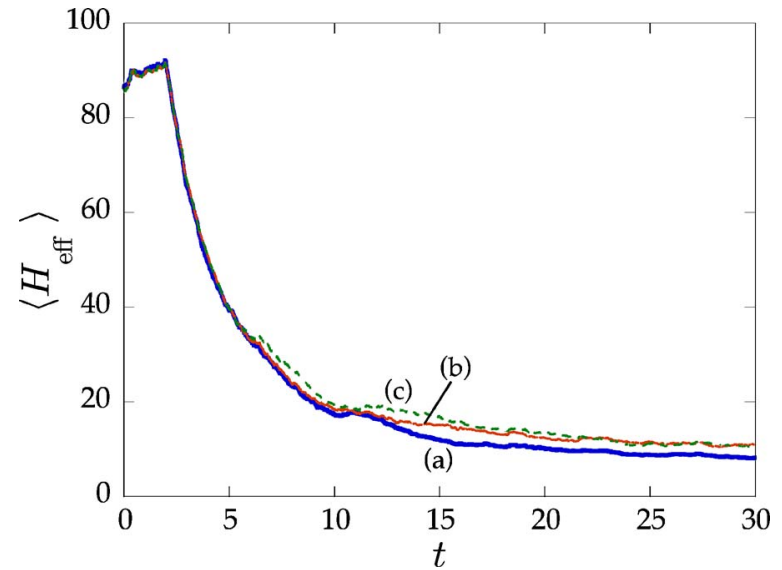

FIG. 13. (Color online) Ensemble energy evolution comparing evolution with and without invoking the adiabatic approximation. Heavy solid line (a): adiabatic approximation. Light solid line (b): full atom-cavity dynamics at $4 \mathrm{GHz}$ detuning from atomic resonance. Dashed line: full atom-cavity dynamics at $2 \mathrm{GHz}$ detuning from atomic resonance. Each curve represents an average over 128 trajectories.

tion. Other ensemble-averaged quantities corroborate this point. For the $4 \mathrm{GHz}$ detuning case, the atomic excited-state population $\left\langle\sigma^{\dagger} \sigma\right\rangle$ is $8.4 \times 10^{-3}$ (corresponding to a spontaneous emission rate of 0.16); the adiabatic-approximation expression $\widetilde{g}^{2}|\alpha|^{2} /\left(\widetilde{\Delta}^{2}+\widetilde{\kappa}^{2}\right)$ predicts a similar value of 8.6 $\times 10^{-3}$ if we include a factor of $1-\left\langle E_{\text {eff }}\right\rangle / 2 V_{\max }=0.98$ to account for the ensemble-averaged spatial distribution of the atoms. For the $2 \mathrm{GHz}$ case, the excited-state population is $3.4 \times 10^{-2}$ (spontaneous emission rate of 0.68 ), agreeing well with the adiabatic prediction of $3.5 \times 10^{-2}$. In both cases, the cavity excitation $\left\langle a^{\dagger} a\right\rangle=0.98$, in good agreement with the adiabatic value of 1 .

\section{CONCLUSION}

Real-time quantum feedback control is worth investigating not only because of potential future applications, but because it will provide an undeniable litmus test of quantum measurement theory at the level of individual measurement records produced by measuring single systems. In this work we have shown that, by using a simplified estimation technique coupled with a relatively simple feedback algorithm, a single atom may be cooled close to its ground state in a realistic optical cavity. We have also explored the effectiveness of the feedback algorithm in various parameter regimes and we hope that this will help to guide future experiments.

\section{ACKNOWLEDGMENTS}

The authors would like to thank Andrew Doherty and Sze Tan for helpful discussions. This research was performed in part using the resources of the Advanced Computing Laboratory, Institutional Computing Initiative, and LDRD program of Los Alamos National Laboratory. 


\section{APPENDIX A: SIMPLE FEEDBACK COOLING AND SQUEEZING}

Here we analyze the effect of the simple centroid-only feedback cooling algorithm described in Sec. III B. This involves switching the optical potential to a high value when the atom is climbing a potential well, and switching it to a low value when it is falling. Recall that the value of the "switched high" field is $\left(1+\varepsilon_{1}\right) E$ and the "switched low" field is $\left(1-\varepsilon_{2}\right) E$, so that the potential height has the corresponding values $\left(1+\varepsilon_{1}\right)^{2} V$ and $\left(1-\varepsilon_{2}\right)^{2} V(E$ and $V$ are the respective unmodulated values).

Since the feedback algorithm involves more than simply driving the system with an external force, to understand the action of the algorithm one must analyze its effect both on the means (the centroid) and the variances of the atomic position and momentum. The effect on the centroid is to damp the motion. In one cooling cycle, the energy of the centroid is multiplied by a "cooling factor"

$$
C_{\mathrm{fb}}=\left(\frac{1-\varepsilon_{2}}{1+\varepsilon_{1}}\right)^{2},
$$

which is valid in the harmonic approximation.

The effect on the variances is, however, to squeeze the atomic wave function. To understand why this is the case, consider what happens when the height of the potential is changed. This changes the frequency of the effective harmonic oscillator governing the atomic motion, and as a consequence, a wave function that was not squeezed in the phase space of the old harmonic oscillator, is squeezed in the space of the new one. In particular, raising the height of the potential scales phase space so that momentum is squeezed, and conversely lowering the potential squeezes position. To determine the rate of squeezing, it is useful to consider what happens in one "cooling cycle." For example, we may define the cooling cycle to start when the atom crosses the bottom of the well, and end when the atom again crosses the bottom of the well traveling in the original direction (essentially one period of oscillation). Examining the effect of each of the changes in the potential during a cooling cycle, we find that the result of a single cycle is to multiply the momentum variance of the atomic wave function by a "magnification factor"

$$
M_{\mathrm{fb}}=\left(\frac{1+\varepsilon_{1}}{1-\varepsilon_{2}}\right)^{2},
$$

in the harmonic approximation. This factor is precisely the inverse of the cooling factor $C_{\mathrm{fb}}$.

This situation is analogous to an attempt to cool a classical ensemble of particles using a control algorithm designed for a single particle; although the algorithm will cool a single particle, it will almost certainly heat the other members of the ensemble. This will be an important consideration in designing an improved feedback algorithm. A simulation of this simple feedback algorithm is given in Fig. 2. This shows that after a few cooling cycles in which the total energy is initially reduced, the resulting squeezing overtakes the cooling of the centroid, and the algorithm actually heats the atomic motion.

\section{APPENDIX B: HEATING MECHANISMS}

Spontaneous emission. Spontaneous emission events kick the atom in both directions, and therefore cause momentum diffusion. The effect of this is to increase the energy, on average, linearly with time. This rate depends both upon the spontaneous-emission rate as well as the magnitude of the associated kicks. One point to note is that the magnitude of each kick is not merely determined by the momentum of the emitted photon, but also by the difference between the momentum of the excited and ground state wave functions. More precisely, we can factor the atomic state vector $|\psi\rangle$ just before a spontaneous-emission event into a product of internal and motional states

$$
|\psi\rangle=\left|\psi_{\mathrm{e}}\right\rangle|\mathrm{e}\rangle+\left|\psi_{\mathrm{g}}\right\rangle|\mathrm{gf}\rangle
$$

where $\left|\psi_{\alpha}\right\rangle$ are states in the center-of-mass space of the atom, and "e" and "g" denote excited and ground atomic energy levels, respectively. Then in an unraveling of the master equation (1) where the spontaneously emitted photons are detected but do not give position information about the atom, the one-dimensional atomic state just after a spontaneousemission event is just the previous state with the internal state lowered and a momentum-recoil factor

$$
|\psi\rangle=\left|\psi_{\mathrm{e}}\right\rangle|\mathrm{g}\rangle e^{-i \tilde{u} X}
$$

where the random variable $\tilde{u}$ is chosen from the projected angular distribution of the resonance fluorescence $N(\widetilde{u})$ referred to in the SME (7). Thus, in addition to the emission momentum recoil, the spontaneous emission effectively converts the atomic state from the ground state wave function to the excited state wave function. In the regime where the adiabatic approximation is valid, we have the relation

$$
\left|\psi_{\mathrm{e}}\right\rangle=\frac{\alpha g}{\Delta} \cos (k x)\left|\psi_{\mathrm{g}}\right\rangle
$$

and thus we can interpret this extra process as being simply another momentum kick due to the absorption of a cavity photon (i.e., a superposition of a photon-recoil kick in either direction along the cavity axis).

The actual rate of spontaneous emissions may be estimated by multiplying the spontaneous emission rate by the average excited state population, giving $\widetilde{\gamma} \widetilde{g}^{2}|\alpha|^{2} /\left(\widetilde{\Delta}^{2}+\widetilde{\kappa}^{2}\right)$ for an atom localized at a field antinode. Calculating this for the conditions that we use in the numerical simulations in the body of the text, we find that the rate is around 0.17 . In the far-detuned regime that we study here, the spontaneousemission heating is a minor effect compared to the other heating mechanisms.

Measurement backaction. The rate of heating from the backaction due to the continuous measurement process may be estimated by examining the effective master equation for the system in Eq. (14). The energy of the atomic motion in the effective potential, defined with respect to the minimum potential energy, is simply 


$$
E_{\mathrm{eff}}=\pi P^{2}+\frac{\pi}{\tilde{k}^{2}}\left[1-\cos ^{2}(\tilde{k} X)\right]
$$

The rate of increase of this energy, averaged over all possible trajectories, due to the measurement is determined by the term in the SME proportional to $\Gamma$, which gives

$$
\begin{aligned}
\partial_{t}\left\langle E_{\mathrm{eff}}\right\rangle_{\text {meas }} & =2 \Gamma \operatorname{Tr}\left\{E_{\mathrm{eff}} \mathcal{D}\left[\cos ^{2}(\tilde{k} x)\right] \rho\right\} \\
& =8 \pi \Gamma\left\langle\cos ^{2}(\tilde{k} X) \sin ^{2}(\tilde{k} X)\right\rangle .
\end{aligned}
$$

Note that the $\Gamma$ here is actually a time-averaged quantity when feedback is applied, which we assume to be well approximated by the unmodulated value of $\Gamma$ in Eq. (17), at least to $O(\varepsilon)$. The simplest limit for this expression is if we assume the particle to be randomly distributed along the potential, which is a reasonably good approximation to the initial heating rate

$$
\partial_{t}\left\langle E_{\mathrm{eff}}\right\rangle_{\mathrm{meas}}=\pi \Gamma \widetilde{k}^{2} .
$$

A more appropriate limit for this heating rate after cooling has taken place is the low-temperature regime, where we can make the harmonic approximation so that the rate becomes

$$
\partial_{t}\left\langle E_{\mathrm{eff}}\right\rangle_{\text {meas }} \approx 8 \Gamma \widetilde{k}^{2}\left\langle\widetilde{k}^{2} X^{2}\right\rangle \approx 4 \Gamma \widetilde{k}^{4}\left\langle E_{\mathrm{eff}}\right\rangle,
$$

which is valid for small $\left\langle E_{\mathrm{eff}}\right\rangle$.

Note that although we attribute this heating to measurement backaction, an equivalent, measurement-independent picture for this heating process is the cavity decay process. Because light escapes the cavity in discrete units at random times, there is a stochastic component to the cavity field intensity. Thus, there is a stochastic, heating force on the atoms. The rate obtained by this argument must be the same as the rate that we just obtained, since the heating rate obtained by averaging over all possible trajectories in a "quantum jump" unraveling of the master equation must be the same as in the "quantum diffusion" unraveling that we use here.

\section{APPENDIX C: EFFECTS OF DETECTION INEFFICIENCY AND FEEDBACK DELAYS}

\section{Detection efficiency}

Now we come to one of the two primary limitations in an experimental implementation of the present system, that of limited efficiency of the optical detectors. As this limits the information incorporated by the estimator, it is natural to expect that the best cooling is achieved for unit efficiency, and that the cooling simply gets worse for inefficient measurements. Simulated final energies using the Gaussian estimator are shown in Fig. 14, which for the most part bear out this expectation. One remarkable feature of these results is that the cooling is extremely robust to detector inefficiency. It is only down around $50 \%$ detection efficiency that a few trajectories begin to pull up the average energies, and below about $35 \%$ efficiency that there is a clear trend towards large steady-state temperatures. This robustness is very encourag-

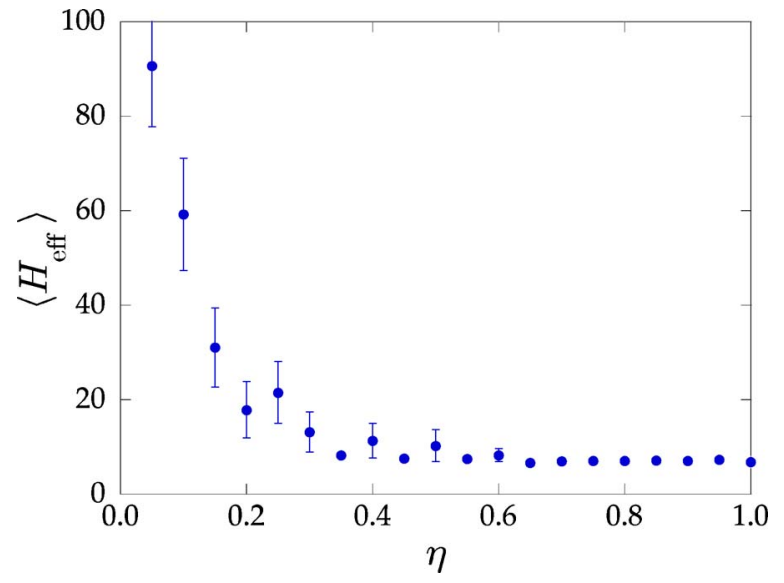

FIG. 14. (Color online) Final energies, measured over the interval from $t=90$ to 100 , as the detection efficiency $\eta$ varies; other parameters match the canonical set. Cooling is according to the improved algorithm based on the Gaussian estimator; error bars reflect standard errors from averages over 128 trajectories.

ing that an experimental demonstration of quantum feedback cooling will work well in spite of imperfect detectors.

\section{Speed and feedback delay issues}

In the simulations we have discussed so far, the Gaussian estimator was updated every time interval of $\Delta t_{G}=0.0005$. For the canonical parameter set, this update interval corresponds to about $3 \mathrm{~ns}$ in physical time. This is obviously a very short time in which to evolve the estimation equations (19) as well as the curve fit outlined in Sec. III B. Therefore, the first issue we will tackle is the speed with which one can iterate the control algorithm.

Curve fit. Recall that, given a set of estimates $y_{\mathrm{est}, n}$ corresponding to times $t_{n}$, we wish to implement the curve fit of the function $a_{0}+a_{1} x+a_{2} x^{2}$ to the last $q$ values of $y_{\mathrm{est}, i}$. The curve-fit coefficients are given by the solution of the normal equations

$$
\left(\begin{array}{ccc}
\Sigma_{0} & \Sigma_{1} & \Sigma_{2} \\
\Sigma_{1} & \Sigma_{2} & \Sigma_{3} \\
\Sigma_{2} & \Sigma_{3} & \Sigma_{4}
\end{array}\right)\left(\begin{array}{l}
a_{0} \\
a_{1} \\
a_{2}
\end{array}\right)=\left(\begin{array}{l}
S_{0} \\
S_{1} \\
S_{2}
\end{array}\right)
$$

where $\Sigma_{n}:=\sum_{j=1}^{q}\left(x_{j}\right)^{n}$ and $S_{n}:=\sum_{j=1}^{q} y_{j}\left(x_{j}\right)^{n}$. However, recomputing the sums in this system of equations at each iteration is not computationally efficient. To make the on-the-fly calculation of this curve fit feasible, we can instead implement the coupled recurrence relations

$$
\begin{gathered}
S_{0}^{(n)}=S_{0}^{(n-1)}+y_{\text {est }, n}+y_{\text {est }, n-m}, \\
S_{1}^{(n)}=S_{1}^{(n-1)}-S_{0}^{(n-1)}+m y_{\text {est }, n-m}, \\
S_{2}^{(n)}=S_{2}^{(n-1)}-2 S_{1}^{(n-1)}+S_{0}^{(n-1)}-m^{2} y_{\text {est }, n-m}
\end{gathered}
$$

and then compute the fitted slope coefficient at time $t_{n}$ according to 


$$
\begin{aligned}
a_{1}= & \frac{18(2 q-1)}{q(q+1)(q+2)}\left(S_{0}+\frac{2(8 q-11)}{3(q-1)(q-2)} S_{1}\right. \\
& \left.+\frac{10}{(q-1)(2 q-1)} S_{2}\right) .
\end{aligned}
$$

For notational convenience, we have defined the times corresponding to the last $q$ measurements to be $t_{n}=1-n$, so that the fitted slope at the current time is simply $a_{1}$. Thus, we can simply trigger the feedback cooling algorithm on the sign of $a_{1}$, switching the potential high or low for a positive or negative sign of $a_{1}$, respectively. The speed of this algorithm is largely independent of the number of samples used in the curve fit, assuming that the table of the last $q$ values can be accessed quickly (i.e., it can fit into fast memory).

Later we will examine the effects of computation and signal propagation delays on the cooling performance. Such delays are easily accounted for in the present algorithm by using the curve fit to extrapolate forward in time. To do this, we require the quadratic fit coefficient

$$
a_{2}=\frac{30}{q(q+1)(q+2)}\left(S_{0}+\frac{6}{q-2} S_{1}+\frac{6}{(q-1)(q-2)} S_{2}\right),
$$

so that we trigger on the value of the current-time slope

$$
a_{1}+2 a_{2} d
$$

where $d$ is the delay in multiples of $\Delta t_{\mathrm{G}}$ from the time of the most recent data used in the fit to the current time. Due to the predictive nature of this fitting scheme, we expect that the cooling should be robust to small delays.

Gaussian estimator. The other issue to be resolved is to maximize the efficiency of updating the Gaussian estimator. One computationally expensive aspect of the form of the equations (19) is the need to evaluate transcendental functions. A substantial speed improvement results from introducing the variables $x_{1}:=\sin \left(2 \tilde{k}\langle X\rangle_{\mathrm{e}}\right), x_{2}:=\cos \left(2 \tilde{k}\langle X\rangle_{\mathrm{e}}\right)$, $x_{3}:=\langle P\rangle_{\mathrm{e}} / \tilde{k}, x_{4}:=2 \widetilde{k}^{2} V_{X}^{\mathrm{e}}, x_{5}:=\exp \left(-2 \widetilde{k}^{2} V_{X}^{\mathrm{e}}\right), x_{6}:=V_{P}^{\mathrm{e}} / \widetilde{k}^{2}$, and $x_{7}:=\exp \left(-2 \widetilde{k}^{2} V_{X}^{\mathrm{e}}\right)$, we can rewrite the estimator equations in the form

$$
\begin{gathered}
d x_{1}=\left(4 \pi \tilde{k}^{2} x_{2} x_{3}-4 \eta \Gamma x_{1}^{3} x_{4}^{2} x_{5}^{2}\right) d t+\sqrt{8 \eta \Gamma} x_{1} x_{2} x_{4} x_{5} d W_{\mathrm{e}} \\
d x_{2}=-\left(4 \pi \widetilde{k}^{2} x_{1} x_{3}+4 \eta \Gamma x_{1}^{2} x_{2} x_{4}^{2} x_{5}^{2}\right) d t-\sqrt{8 \eta \Gamma} x_{1}^{2} x_{4} x_{5} d W_{\mathrm{e}} \\
d x_{3}=-V_{\max } x_{1} x_{5} d t+\sqrt{8 \eta \Gamma} x_{1} x_{5} x_{7} d W_{\mathrm{e}} \\
d x_{4}=\left(8 \pi \widetilde{k}^{2} x_{7}-4 \eta \Gamma x_{1}^{2} x_{4}^{2} x_{5}^{2}\right) d t+\sqrt{8 \eta \Gamma} x_{2} x_{4}^{2} x_{5} d W_{\mathrm{e}} \\
d x_{5}=\left[-8 \pi \widetilde{k}^{2} x_{5} x_{7}+4 \eta \Gamma\left(x_{1}^{2} x_{5}-x_{2}^{2} x_{4}^{2}\right) x_{4}^{2} x_{5}^{2}\right] d t \\
-\sqrt{8 \eta \Gamma} x_{2} x_{4}^{2} x_{5}^{2} d W_{\mathrm{e}}
\end{gathered}
$$

$$
\begin{gathered}
d x_{6}=\left(-4 V_{\max } x_{2} x_{5} x_{7}+\Gamma\left[1+x_{5}^{4}\left(1-2 x_{2}^{2}\right)\right]-8 \eta \Gamma x_{1}^{2} x_{5}^{2} x_{7}^{2}\right) d t \\
-\sqrt{2 \eta \Gamma}\left[1-2\left(x_{4}+2 x_{7}^{2}\right) x_{5}\right] x_{2} d W_{\mathrm{e}}, \\
d x_{7}=\left(2 \pi \widetilde{k}^{2} x_{6}-V_{\max } x_{2} x_{4} x_{5}-4 \eta \Gamma x_{1}^{2} x_{4} x_{5}^{2} x_{7}\right) d t \\
+\sqrt{8 \eta \Gamma} x_{2} x_{4} x_{5} x_{7} d W_{\mathrm{e}},
\end{gathered}
$$

with

$$
d W_{\mathrm{e}}=\frac{d r}{\sqrt{\eta \widetilde{\kappa}}}+\sqrt{2 \eta \Gamma}\left(1+x_{2} x_{5}\right) d t
$$

thus obviating any need for transcendental-function evaluations. However, it turns out that the evolution of these equations via the Euler method is much less stable than the evolution of the original equations. This is due to the fact that there are pairs of variables $\left[\left(x_{1}, x_{2}\right)\right.$ and $\left.\left(x_{4}, x_{5}\right)\right]$ that evolve separately but must satisfy consistency conditions $\left[x_{1}^{2}+x_{2}^{2}\right.$ $=1$ and $x_{5}=\exp \left(-x_{4}\right)$ ]; also a large stochastic fluctuation can push these values into unphysical ranges (e.g., $x_{1}>1$ ). A better strategy is to emulate the results of an Euler solution to the original estimator equations, and hence update $x_{1}, x_{2}$, and $x_{5}$ using the increments

$$
\begin{gathered}
\Delta x_{1}=x_{1}\left[\cos \left(2 \tilde{k} \Delta\langle X\rangle_{\mathrm{e}}\right)-1\right]+x_{2} \sin \left(2 \tilde{k} \Delta\langle X\rangle_{\mathrm{e}}\right), \\
\Delta x_{2}=x_{2}\left[\cos \left(2 \tilde{k} \Delta\langle X\rangle_{\mathrm{e}}\right)-1\right]-x_{1} \sin \left(2 \tilde{k} \Delta\langle X\rangle_{\mathrm{e}}\right), \\
\Delta x_{5}=x_{5} \exp \left(-\Delta x_{4}\right),
\end{gathered}
$$

where

$$
2 \tilde{k} \Delta\langle X\rangle_{\mathrm{e}}=4 \pi \widetilde{k}^{2} x_{3} \Delta t_{\mathrm{G}}+\sqrt{8 \eta \Gamma} x_{1} x_{4} x_{5} \Delta W_{\mathrm{e}},
$$

$\Delta W_{\mathrm{e}}(t):=\int_{t}^{t+\Delta t_{\mathrm{G}}} d W_{\mathrm{e}}$, and $\Delta x_{4}$ is the Euler-update increment from Eqs. (C6). These equations can then be expanded in powers of $2 \tilde{k} \Delta\langle X\rangle_{\mathrm{e}}$ to avoid computing the transcendental functions. A final trick to help stabilize the equations is to multiply the $x_{1}$ and $x_{2}$ variables at each time step by the normalization quantity $\left[1-\left(x_{1}^{2}+x_{2}^{2}\right)\right] / 2$, which explicitly maintains the identity $x_{1}^{2}+x_{2}^{2}=1$ without requiring the evaluation of a square root.

Execution times. To evaluate how quickly the control algorithm can be iterated, we implemented the control algorithm on a modern, general-purpose, serial microprocessor (1.25 GHz Alpha EV68). A single iteration of the estimator according to Eqs. (19) and a curve fit, including all steps necessary to compute the control output, can be completed in about 260 ns. Switching to the faster method of Eqs. (C6) with the more stable updates (C8) can be completed in $140 \mathrm{~ns}$ with a truncation at fourth order in $2 \tilde{k} \Delta\langle X\rangle_{\mathrm{e}}$, and $120 \mathrm{~ns}$ with a second-order truncation (including a normalization step). The cooling performance is essentially the same with all these algorithms, in that they all reach the final temperature; the exception is an algorithm that uses Eqs. (C6) without the modifications of Eqs. (C8), which has substantially worse cooling performance. The tradeoff in the truncated-expansion algorithms is that lower-order trunca- 


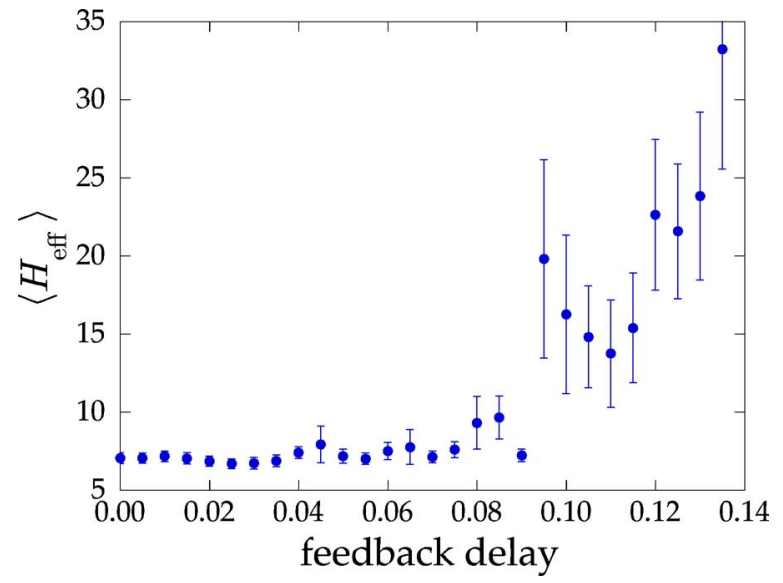

FIG. 15. (Color online) Final energies, measured over the interval from $t=90$ to 100 , as a function of the feedback delay $\tau_{d}$. (Time is measured in units of the $5.8 \mu \mathrm{s}$ harmonic-oscillator period.) Cooling is according to the improved algorithm based on the Gaussian estimator, with extrapolation implemented in the curve fit; error bars reflect standard errors from averages over 128 trajectories. Other parameters are as in the canonical set.

tions are faster but also more unstable and thus require more resets of the estimator.

Based on this performance, and assuming Moore's law, general-purpose hardware will not be able to iterate this algorithm in $3 \mathrm{~ns}$ for about a decade. However, even presently available, specialized technologies such as multicore processors and field-programmable gate arrays (FPGAs) can likely be used to significantly decrease the execution time. For example, because the curve fit can be moved onto a separate logical processor, the estimator can be updated more quickly (84 ns for the fourth-order version, $70 \mathrm{~ns}$ for the secondorder version); the extra 60 ns required to generate the feedback value via the curve fit then represents an additional delay, but does not limit the rate at which the estimator can be iterated. Further parallelization should bring these times down substantially.

Impact on cooling performance. Given these limitations in computing power it is necessary to evaluate how quickly the algorithm needs to be iterated in order to achieve good cooling performance. The cooling performance in the presence of feedback delay $\tau_{\mathrm{d}}=d \Delta t_{\mathrm{G}}$ is shown in Fig. 15. The cooling is very robust to the delay, since we can compensate by extrapolating the curve fit. The cooling behavior is essentially unaffected out to a delay of about $460 \mathrm{~ns}$, which is well above the $60 \mathrm{~ns}$ figure that we mentioned for generating the feedback value, and thus additional signal propagation and processing delays should not be too problematic.

Limitations on the time $\Delta t_{\mathrm{G}}$ required to iterate the Gaussian estimator can also seriously impact the cooling performance. Not only does a large $\Delta t_{\mathrm{G}}$ step reduce the quality of the estimated solution, but in fitting the quadratic curve over a finite interval, there are fewer points involved in the fit, reducing the effectiveness of the fit's noise reduction. These effects on the cooling behavior are illustrated in Fig. 16, which shows that the late-time ensemble energies grow approximately linearly with $\Delta t_{\mathrm{G}}$. Here, the 70 ns figure mentioned above for $\Delta t_{\mathrm{G}}$ yields a final energy of around 25 ,

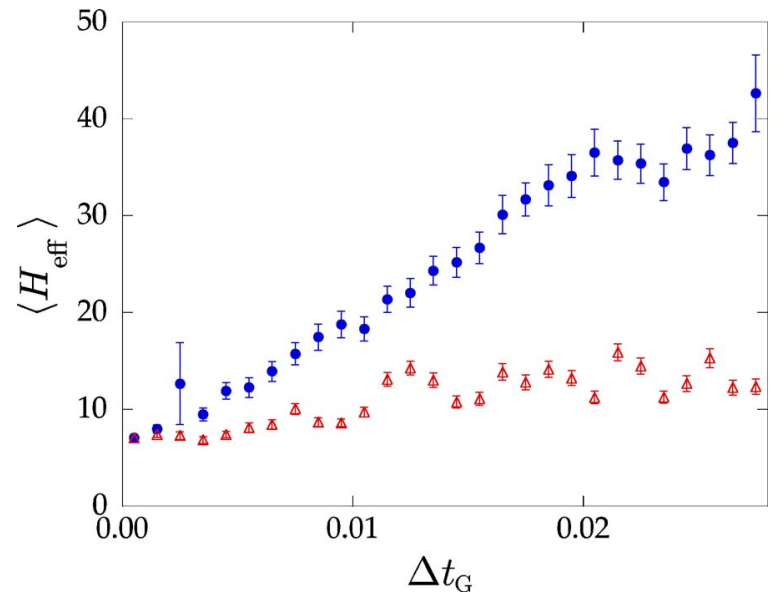

FIG. 16. (Color online) Final energies, measured over the interval from $t=90$ to 100 , as a function of the Gaussian estimator time step $\Delta t_{\mathrm{G}}$. (Time is measured in units of the $5.8 \mu \mathrm{s}$ harmonicoscillator period.) Shown are results obtained by reducing the number of samples to keep the time interval for the quadratic curve constant (circles) as well as results obtained via the multipleestimator staggering method to maintain a 300-point fit (triangles). Error bars reflect standard errors from averages over 128 trajectories. Other parameters are as in the canonical set.

compared to around 9 for the smallest value of $\Delta t_{\mathrm{G}}$ $=0.0005$ ( $3 \mathrm{~ns})$ shown, a substantial decrease in the cooling effectiveness. One possible way to address this problem is to use multiple Gaussian estimators that are staggered in time. For example, defining $\Delta t_{\min }=0.0005$, and given that the computational hardware is limited to $\Delta t_{\mathrm{G}}=N \Delta t_{\text {min }}$ for some integer $N$, we can arrange to have $N$ independent implementations of the Gaussian estimator executing in parallel. They all receive the same measurement record as input, but they are staggered in time such that one produces output at each small time step $\Delta t_{\min }=0.0005$. In this way, each of the individual

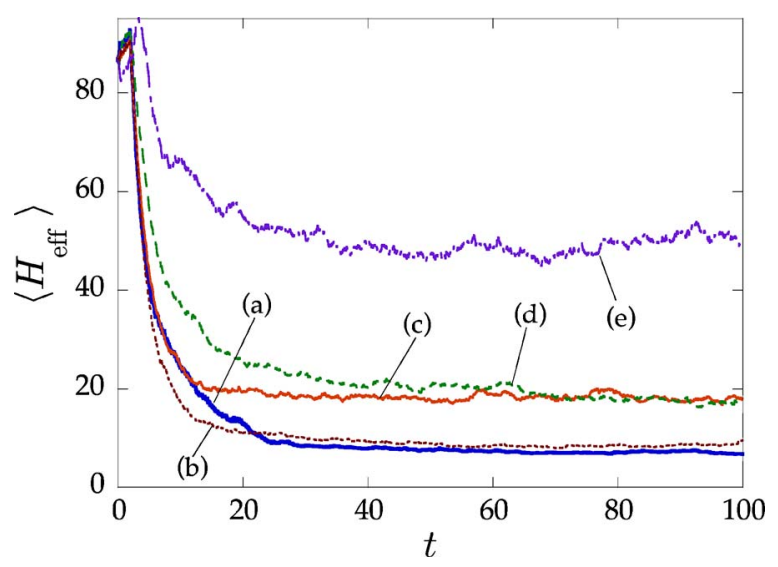

FIG. 17. (Color online) Ensemble energy evolution illustrating the combined effects of detection efficiency $\eta$, feedback delay $\tau_{d}$, and estimator time step $\Delta t_{\mathrm{G}}$. (Time is measured in units of the $5.8 \mu$ s harmonic-oscillator period.) These parameter values for the curves (a)-(d) are given in the text; others are as in the canonical set. Extrapolation is implemented in the curve fit, and multipleestimator staggering is used to maintain a 300-point fit. Each curve represents an average over 128 trajectories. 
solutions is of lower quality than a fine-time-step solution, but the curve fit can be fit to all of them (restoring a full 300 point curve fit), thus helping to stabilize the cooling algorithm. The implementation of this method is also displayed in Fig. 16, which shows that this method restores much of the quality of the cooling that was lost in using the simpler algorithm. The rightmost point in this figure corresponds to operating 55 parallel estimators.

So far, we have studied the performance impact on cooling of each of the three equipment-limitation effects (detection efficiency, feedback delay, and estimator time step) separately. Some representative examples of their combined effect are shown in Fig. 17. The four curves, in order of increasing departure from the ideal case, correspond to the following parameters: (a) $\eta=1, \tau_{\mathrm{d}}=0, \Delta t_{\mathrm{G}}=\Delta t_{\min }=0.0005$ (i.e., $3 \mathrm{~ns}$ in physical time); (b) $\eta=0.8, \tau_{\mathrm{d}}=\Delta t_{\mathrm{G}}=10 \Delta t_{\text {min }}$ $=0.005$ (i.e., $29 \mathrm{~ns}$ ); (c) $\eta=0.7, \tau_{\mathrm{d}}=\Delta t_{\mathrm{G}}=25 \Delta t_{\min }=0.0125$ (i.e., $72 \mathrm{~ns}$ ); (d) $\eta=0.5, \quad \tau_{\mathrm{d}}=\Delta t_{\mathrm{G}}=40 \Delta t_{\min }=0.02 \quad$ (i.e., $116 \mathrm{~ns}$ ); (e) $\eta=0.3, \tau_{\mathrm{d}}=\Delta t_{\mathrm{G}}=50 \Delta t_{\min }=0.025$ (i.e., $145 \mathrm{~ns}$ ). The sums of the populations in the lowest two bands for these cases, computed between $t=90$ and 100 , are $(94 \pm 5) \%$, $(84 \pm 5) \%,(45 \pm 4) \%,(47 \pm 4) \%$, and $(13 \pm 2) \%$, respectively. The cooling performance, even for the case that should be within reach of current technology (d), is not unreasonable in comparison to the ideal case (a), although cooling quickly becomes worse as these parameter values are relaxed (e). Even with these effects, this method is certainly suitable for long-time storage of atoms in the optical potential and can still reach the ground state with serviceable efficiency.
[1] Quantum Theory and Measurement, edited by J. A. Wheeler and W. H. Zurek (Princeton University Press, Princeton, 1983).

[2] G. J. Milburn, K. Jacobs, and D. F. Walls, Phys. Rev. A 50, 5256 (1994).

[3] H. Mabuchi, J. Ye, and H. J. Kimble, Appl. Phys. B: Lasers Opt. 68, 1095 (1999).

[4] P. Münstermann, T. Fischer, P. Maunz, P. W. H. Pinkse, and G. Rempe, Phys. Rev. Lett. 82, 3791 (1999).

[5] C. J. Hood, T. W. Lynn, A. C. Doherty, A. S. Parkins, and H. J. Kimble, Science 287, 1447 (2000).

[6] P. W. H. Pinkse, T. Fischer, P. Maunz, and G. Rempe, Nature (London) 404, 365 (2000).

[7] V. P. Belavkin, Autom. Remote Control (Engl. Transl.) 44, 178 (1983).

[8] V. P. Belavkin, in Information Complexity and Control in Quantum Physics, edited by A. Blaquière, S. Diner, and G. Lochak (Springer-Verlag, New York, 1987).

[9] J. A. Sauer, K. M. Fortier, M. S. Chang, C. D. Hamley, and M. S. Chapman, Phys. Rev. A 69, 051804(R) (2004).

[10] M. G. Raizen, J. Koga, B. Sundaram, Y. Kishimoto, H. Takuma, and T. Tajima, Phys. Rev. A 58, 4757 (1998).

[11] V. P. Belavkin, Rep. Math. Phys. 43, 405 (1999).

[12] A. C. Doherty and K. Jacobs, Phys. Rev. A 60, 2700 (1999).

[13] A. C. Doherty, S. Habib, K. Jacobs, H. Mabuchi, and S. M. Tan, Phys. Rev. A 62, 012105 (2000).

[14] M. A. Armen, J. K. Au, J. K. Stockton, A. C. Doherty, and H. Mabuchi, Phys. Rev. Lett. 89, 133602 (2002).

[15] T. Fischer, P. Maunz, P. W. H. Pinkse, T. Puppe, and G. Rempe, Phys. Rev. Lett. 88, 163002 (2002).

[16] L. R. D. Vitali, S. Mancini, and P. Tombesi, J. Opt. Soc. Am. B 20, 1054 (2003).

[17] A. Hopkins, K. Jacobs, S. Habib, and K. Schwab, Phys. Rev. B 68, 235328 (2003).

[18] J. Geremia, J. K. Stockton, and H. Mabuchi, e-print quant-ph/ 0309034.

[19] J. Geremia, J. K. Stockton, and H. Mabuchi, Science 304, 270 (2004).

[20] R. van Handel, J. K. Stockton, and H. Mabuchi, IEEE Trans. Autom. Control 50, 768 (2005).

[21] J. Geremia, J. K. Stockton, and H. Mabuchi, Phys. Rev. Lett.
94, 203002 (2005).

[22] J. Geremia, J. K. Stockton, and H. Mabuchi, e-print quant-ph/ 0501033.

[23] G. A. Smith, S. Chaudhury, A. Silberfarb, I. H. Deutsch, and P. S. Jessen, Phys. Rev. Lett. 93, 163602 (2004).

[24] W. P. Smith, J. E. Reiner, L. A. Orozco, S. Kuhr, and H. M. Wiseman, Phys. Rev. Lett. 89, 133601 (2002).

[25] M. R. James, Phys. Rev. A 69, 032108 (2004).

[26] J. E. Reiner, W. P. Smith, L. A. Orozco, H. M. Wiseman, and J. Gambetta, Phys. Rev. A 70, 023819 (2004).

[27] J. Hanssen, V. Milner, T. Meyrath, and M. G. Raizen (unpublished).

[28] N. V. Morrow, S. K. Dutta, and G. Raithel, Phys. Rev. Lett. 88, 093003 (2002).

[29] V. Vuletić, A. T. Black, and J. K. Thompson, e-print quant-ph/ 0410168.

[30] D. A. Steck, K. Jacobs, H. Mabuchi, T. Bhattacharya, and S. Habib, Phys. Rev. Lett. 92, 223004 (2004).

[31] V. Steixner, P. Rabl, and P. Zoller, e-print quant-ph/0506187.

[32] P. Rabl, V. Steixner, and P. Zoller, e-print quant-ph/0506185.

[33] H. M. Wiseman and A. C. Doherty, Phys. Rev. Lett. 94, 070405 (2005).

[34] P. Horak, G. Hechenblaikner, K. M. Gheri, H. Stecher, and H. Ritsch, Phys. Rev. Lett. 79, 4974 (1997).

[35] W. Alge, K. Ellinger, H. Stecher, K. M. Gheri, and H. Ritsch, Europhys. Lett. 39, 491 (1997).

[36] M. Gangl and H. Ritsch, Phys. Rev. A 61, 011402(R) (2000).

[37] M. Gangl and H. Ritsch, Phys. Rev. A 61, 043405 (2000).

[38] V. Vuletić and S. Chu, Phys. Rev. Lett. 84, 3787 (2000).

[39] P. Domokos, P. Horak, and H. Ritsch, J. Phys. B 34, 187 (2001)

[40] M. Gangl and H. Ritsch, Phys. Rev. A 64, 063414 (2001).

[41] P. Horak and H. Ritsch, Phys. Rev. A 64, 033422 (2001).

[42] S. J. van Enk, J. McKeever, H. J. Kimble, and J. Ye, Phys. Rev. A 64, 013407 (2001).

[43] P. Maunz, T. Puppe, I. Schuster, N. Syassen, P. W. H. Pinkse, and G. Rempe, Nature (London) 428, 50 (2004).

[44] V. P. Belavkin, in Modelling and Control of Systems, edited by A. Blaquière (Springer, New York, 1988).

[45] V. P. Belavkin, Phys. Lett. A 140, 355 (1989). 
[46] D. Chruśeiński and P. Staszewski, Phys. Scr. 45, 193 (1992).

[47] M. D. Srinivas and E. B. Davies, Opt. Acta 28, 981 (1981).

[48] A. Barchielli, L. Lanz, and G. M. Prosperi, Opt. Acta 13, 779 (1983).

[49] A. Barchielli and G. Lupieri, J. Math. Phys. 26, 2222 (1985).

[50] L. Diosi, Phys. Lett. 114A, 451 (1986).

[51] L. Diosi, Phys. Lett. A 129, 419 (1986).

[52] N. Gisin, Phys. Rev. Lett. 52, 1657 (1984).

[53] C. M. Caves and G. J. Milburn, Phys. Rev. A 36, 5543 (1987).

[54] Barchielli, Int. J. Theor. Phys. 32, 2221 (1993).

[55] H. Carmichael, An Open Systems Approach to Quantum Optics (Springer-Verlag, Berlin, 1993).

[56] H. M. Wiseman and G. J. Milburn, Phys. Rev. A 47, 642 (1993).

[57] O. L. R. Jacobs, Introduction to Control Theory (Oxford University Press, Oxford, 1993).

[58] P. S. Maybeck, Stochastic Models, Estimation, and Control (Wiley, Chichester, 1996).

[59] P. Whittle, Optimal Control: Basics and Beyond (Academic Press, New York, 1982).

[60] K. Zhou, J. C. Doyle, and K. Glover, Robust and Optimal Control (Prentice Hall, Englewood Cliffs, NJ, 1995).

[61] H. M. Wiseman, S. Mancini, and J. Wang, Phys. Rev. A 66, 013807 (2002).

[62] K. Jacobs, Phys. Rev. A 67, 030301(R) (2003).
[63] M. J. Holland, D. F. Walls, and P. Zoller, Phys. Rev. Lett. 67, 1716 (1991).

[64] P. Storey, M. Collett, and D. Walls, Phys. Rev. Lett. 68, 472 (1992).

[65] R. Quadt, M. Collett, and D. F. Walls, Phys. Rev. Lett. 74, 351 (1995).

[66] A. C. Doherty, A. S. Parkins, S. M. Tan, and D. F. Walls, Phys. Rev. A 57, 4804 (1998).

[67] The terms in the stochastic master equation that describe homodyne detection are derived in Ref. [56]. The term that describes spontaneous emission is given in, e.g., Refs. [73,74].

[68] V. P. Belavkin (unpublished).

[69] H. M. Wiseman, Ph.D. thesis, University of Queensland, Brisbane, 1994.

[70] C. J. Hood, M. S. Chapman, T. W. Lynn, and H. J. Kimble, Phys. Rev. Lett. 80, 4157 (1998).

[71] P. E. Kloeden and E. Platen, Numerical Solution of Stochastic Differential Equations (Springer, Berlin, 1992).

[72] W. H. Zurek, S. Habib, and J. P. Paz, Phys. Rev. Lett. 70, 1187 (1993).

[73] K. A. Jacobs, M. J. Collett, H. M. Wiseman, S. M. Tan, and D. F. Walls, Phys. Rev. A 54, 2260 (1996).

[74] P. Marte, R. Dum, R. Taïeb, P. Zoller, M. S. Shahriar, and M. Prentiss, Phys. Rev. A 49, 4826 (1994). 